Akhavan-Tafti Mojtaba (Orcid ID: 0000-0003-3721-2114)

Slavin James A. (Orcid ID: 0000-0002-9206-724X)

Eastwood Jonathan P. (Orcid ID: 0000-0003-4733-8319)

Cassak Paul A (Orcid ID: 0000-0002-5938-1050)

Gershman Daniel J (Orcid ID: 0000-0003-1304-4769)

\title{
MMS Multi-Point Analysis of FTE Evolution: Physical Characteristics and Dynamics
}

\author{
M. Akhavan-Tafti ${ }^{1}$, J. A. Slavin ${ }^{1}$, J. P. Eastwood ${ }^{2}$, P. A. Cassak ${ }^{3}$, D. J. Gershman ${ }^{4}$ \\ ${ }^{1}$ Climate and Space Sciences and Engineering, University of Michigan, Ann Arbor, MI, USA. \\ ${ }^{2}$ Blackett Laboratory, Imperial College London, London, UK. \\ ${ }^{3}$ West Virginia University Morgantown, WV, USA. \\ ${ }^{4}$ NASA Goddard Space Flight Center, Greenbelt, MD, USA.
}

Corresponding author: Mojtaba Akhavan-Tafti (akhavant@umich.edu)

Key Points:

This is the author manuscript accepted for publication and has undergone full peer review but has not been through the copyediting, typesetting, pagination and proofreading process, which may lead to differences between this version and the Version of Record. Please cite this article as doi: 10.1029/2018JA026311

This article is protected by copyright. All rights reserved. 
- The forces inside FTEs observed by MMS suggest plasma acceleration toward and along the FTE's central axis causing plasma to escape

- The roles of adiabatic expansion and reconnection in FTE growth are explored using MMS observations

- The observed sub-adiabatic decrease of plasma pressure as FTE size increases requires plasma heating mechanisms such as reconnection 


\begin{abstract}
Previous studies have indicated that Flux Transfer Events (FTEs) grow as they convect away from the reconnection site along the magnetopause. This increase in FTE diameter may occur via adiabatic expansion in response to decreasing external pressure away from the subsolar region or due to a continuous supply of magnetic flux and plasma to the FTEs' outer layers by magnetic reconnection. Here, we investigate an ensemble of 55 FTEs at the subsolar magnetopause using Magnetospheric Multiscale (MMS) multi-point measurements. The FTEs are initially modeled as quasi-force-free flux ropes in order to infer their geometry and the spacecraft trajectory relative to their central axis. The MMS observations reveal a radiallyinward net force at the outer layers of FTEs which can accelerate plasmas and fields toward the FTE's core region. Inside the FTEs, near the central axis, plasma density is found to decrease as the axial net force increases. It is interpreted that the axial net force accelerates plasmas along the axis in the region of compressing field lines. Statistical analysis of the MMS observations of the 55 FTEs indicates that plasma pressure, $P_{t h}$, decreases with increasing FTE diameter, $\lambda$, as

$\mathrm{P}_{t h, o b s v} \propto \lambda^{-0.24}$. Assuming that all 55 FTEs started out with similar diameters, this rate of plasma pressure decrease with increasing FTE diameter is at least an order of magnitude slower than the theoretical rate for adiabatic expansion (i.e., $P_{\text {th adiab. }} \propto \lambda^{-3.3}$ ), suggesting the presence of efficient plasma heating mechanisms, such as magnetic reconnection, to facilitate FTE growth.
\end{abstract}

\title{
1 Introduction
}

Magnetic reconnection is the process by which topological rearrangement of field lines relieves stress in magnetized plasmas (e.g., Biskamp, 1996; Yamada et al., 2010a; Gonzalez \& Parker, 2016). Magnetic flux ropes are a crucial component of magnetic reconnection (Drake et al., 2006; Daughton et al., 2006). At the Earth's magnetopause, they take the form of FTEs (Russell \& Elphic, 1978; Lee \& Fu, 1985; Scholer, 1988; Eastwood et al., 2007). Theory suggests that FTEs can begin as small (electron- or ion-scale) flux ropes generated inside the magnetopause current layer as a result of multiple X-line reconnection (e.g., Lee \& Fu, 1985; Deng et al., 2004; Daughton et al., 2006; Chen et al., 2008; Daughton et al., 2011).

Kinetic simulations and in-situ observations have suggested that, upon generation, flux ropes in three dimensions evolve in response to their environment moving tailward with the magnetosheath flow and expanding as the external pressure relaxes toward solar wind values (e.g., Berchem \& Russell, 1984; Rijnbeek et al., 1984; Zhao et al., 2016; Farrugia et al., 2016; Teh et al., 2017) and coalescence (e.g., Finn \& Kaw, 1977; Pritchett, 1992; Karimabadi et al., 2011; Song et al., 2012; Zhou et al., 2014; Wang et al., 2017).

Global magnetohydrodynamic (MHD), kinetic, and Vlasiator simulations indicate that subsolar FTEs tend to grow larger as they travel away from the reconnection site along the 
magnetopause (Omidi et al., 2006; Raeder, 2006; Omidi \& Sibeck, 2007; Dorelli \& Bhattacharjee, 2009; Hoilijoki et al., 2017; Akhavan-Tafti et al., 2018; Hoilijoki et al., 2019). The growth slows down when the FTEs approach their lowest energy state (i.e., 'force-free'; Eastwood et al., 2012). The present study seeks to distinguish between FTE 'expansion' and 'growth'. FTE 'expansion' is a type of FTE 'growth' (i.e., increase in diameter) in which magnetic flux remains unchanged throughout the process. Figure 1 illustrates three potential FTE growth mechanisms: i) adiabatic expansion, ii) continuous reconnection, and iii) coalescence. The concentric circles show magnetic islands which are two-dimensional projections of flux ropes. The shading of the islands determine the thermal pressure $p$ inside them with the darkest shades indicating the region of largest $p$. The number of circles per area indicates magnetic field magnitude and the gradient shading inside the islands shows plasma pressure variability across the structure. The number of black circles represents the islands' magnetic flux content $\psi$. The $\mathrm{X}$ markers indicate $\mathrm{X}$-points at which magnetic field lines reconnect. Due to reconnection, plasma is often heated resulting in an enhanced thermal pressure, i.e., darker shades, and increased magnetic flux content, i.e., more circles. Evolution of the islands is represented with increasing time, $t_{1}>t_{2}$; where subscripts 1 and 2 indicate earlier and later evolution stages, respectively.

In the first scenario, in panel $a$, FTEs grow due to pressure-imbalance across the FTE boundary. As the FTE is carried away from the subsolar region, the external magnetosheath pressure will reduce resulting in the temporal expansion of the FTE. Inside these expanding FTEs, plasma pressure will drop but the magnetic flux content remains constant with time $(\psi=B A$; where $\psi$ and $A$ denote the magnetic flux content and the cross-sectional area of an FTE, respectively). 
In the other two scenarios in Figure 1, FTEs also grow via reconnection. In one scenario, see Figure 1 $b$, multiple $\mathrm{X}$-line reconnection may continue after the initial formation of the magnetic island (Lee \& Fu, 1985, 1986). Multiple X-line reconnection may or may not be a steady process (Phan et al., 2007). At $\mathrm{t}=\mathrm{t}_{1}$, the reconnected magnetic field lines form magnetic islands. But, due to the ongoing magnetic reconnection (Raeder, 2006; Hoilijoki et al., 2019), the magnetic islands grow to a size much larger than the original current sheet thickness. During this growth process, plasma is heated due to reconnection (i.e., $p_{2}>p_{1}$ ). Similarly, ongoing reconnection contributes additional magnetic flux to the original magnetic island (i.e., $\psi_{2}>\psi_{1}$ ).

In the other scenario, depicted in Figure 1c, the magnetic islands grow via coalescence. Chains of ion-scale FTEs (Milan et al., 2000; Slavin et al., 2012; Dong et al., 2017; Teh et al., 2017; Wang et al., 2017; Hwang et al., 2018) are formed at the dayside magnetopause due to multiple X-line reconnection (Lee \& $F u, 1985)$. In this scenario, the neighboring FTEs are forced to merge, reconnect, and grow into larger structures, a process called 'coalescence' (Finn \& Kaw, 1977; Pritchett \& Wu, 1979; Biskamp, 1982). Some in-situ MMS studies have found evidence for FTE coalescence at the magnetopause (Alm et al., 2017; Zhou et al., 2017; Wang et al., 2017).

As shown in Figure 1c, repeated formation and convection of magnetic flux ropes during the multiple reconnection process will sometimes force neighboring FTEs to interact and merge via secondary reconnection. This interaction results in heating plasma (i.e., $p_{2}>p+p^{\prime}$; where $p$ and $p$ ' represent plasma pressures of individual magnetic islands) and has been the subject of various simulation studies (e.g., Ding et al., 1992 and references therein). In two dimensions (i.e., antiparallel reconnection), theory suggests that magnetic flux in the two magnetic islands do not add during the coalescence process, $\psi_{2}<\psi+\psi^{\prime}$, where $\psi$ and $\psi^{\prime}$ represent the flux contents of the two magnetic islands. Rather, the flux in the smaller structure merges with the larger one (Fermo et al., 2011). In this manner, magnetic flux tubes from the smaller magnetic island connect on both ends to the flux tubes in the larger magnetic island, and in doing so create a larger magnetic island with a greater volume and a larger total mass of plasma.

The three-dimensional picture of coalescing FTEs is more complex (e.g., Yamada et al., 1990). Magnetic helicity, a measure of twist of magnetic field lines inside flux ropes, influences the coalescence process. In particular, the merging FTEs' shear angle, which is controlled by the axial component of their magnetic fields, will determine the rate and the extent to which FTEs coalesce. Correspondingly, the resulting FTE will contain equal, $\psi_{2}=\psi<\psi_{1}$, in the case of antiparallel reconnection (e.g., Tian et al., 2010), or different magnetic flux than either of the two original FTEs, $\psi-\psi^{\prime} \leq \psi_{2} \leq \psi+\psi^{\prime}$, in the case of component or guide-field reconnection (e.g., Pritchett \& Coroniti, 2004; Furno et al., 2005; R. Wang et al., 2016; Stanier et al., 2017).

The high temporal and spatial resolution MMS observations enable detailed, multi-point investigations of magnetic reconnection. Here, we use a database of 55 previously identified FTEs (Akhavan-Tafti et al., 2018) to investigate the structure and temporal evolution of FTEs. 
The previous study used the constant-alpha force-free model to determine the impact parameter, which is defined as the relative spacecraft distance to the central axis of the flux rope at the closest approach, as well as the radius, core magnetic field intensity, and magnetic flux content of the FTEs. They found that the FTE size distribution followed an exponential law. This result was interpreted as evidence for the plasmoid instability for the onset of fast reconnection. In space and astrophysical environments highly-elongated current sheets are subject to a 'plasmoid instability' that leads to their breakup and gives rise to an increase in the reconnection rate (collisional plasma regime: Dieter Biskamp, 1986; Loureiro et al., 2007, 2012, 2013; Bhattacharjee et al., 2009; Cassak et al., 2009; W. Daughton et al., 2009; Y.-M. Huang \& Bhattacharjee, 2010; Y.-M. Huang et al., 2011; Murphy et al., 2013; Ni et al., 2015; L. Comisso et al., 2015; L Comisso et al., 2016; Luca Comisso \& Grasso, 2016; and collision-less plasma regime: William Daughton et al., 2006; Retinò et al., 2008; W Daughton et al., 2011; Baalrud et al., 2012; Y.-M. Huang \& Bhattacharjee, 2013).

FTEs subjected to an external and/or internal driving source evolve dynamically with time. During this evolution, the physical parameters, which characterize the state of the system, become a function of time. Each FTE is observed at a different point in its evolution, and thus the dynamics of growth and expansion can be studied on a statistical basis. In section 3.1, the cross-sectional profiles of the magnetic and plasma characteristics of the 55 FTEs are investigated. Inherently, no forces exist inside perfectly 'force-free' structures (i.e., $\mathbf{J} \times \mathbf{B} \sim \nabla \mathbf{P} \sim 0$ ). However, residual magnetic and thermal forces are expected inside 'quasiforce-free' FTEs described here by a goodness of fit (i.e., $\chi^{2} \leq 0.1$; Akhavan-Tafti et al., 2018). In fact, the residual forces may contribute to FTEs' temporal evolution. In Section 3.2, the MHD forces are investigated inside the quasi-force-free FTEs. In order to seek evidence for FTE growth and the underlying mechanisms, we evaluate the stress balance inside of these FTE-type flux ropes and their pressure balance with the surrounding magnetosheath. the thermodynamics of FTE growth is studied. In Section 3.3, the force-imbalance across the FTE boundary and the extent to which it may contribute to the expansion of FTEs is discussed. Adiabatic expansion of flux ropes, as suggested by Kumar \& Rust (1996), requires plasma pressure inside ion-scale FTEs to drop rapidly with increasing FTE size. However, reconnection slows the rate at which plasma pressure drops with increasing FTE diameter via plasma heating. We conclude that FTE growth in the subsolar region is most likely driven by continuous reconnection at adjacent Xlines and coalescence. This result is important because it underpins the interplay between FTE evolution and adiabatic (e.g., betatron acceleration) and non-adiabatic (e.g., reconnection, turbulence, wave-particle interaction, etc.) energization of plasmas at the magnetopause (e.g., Hwang et al., 2016).

\section{Experimental Approach}

2.1 Methods 
Subsolar FTEs are selected from 55 near-equatorial orbital passes (11/03/2015 — 12/28/2015). High spatial and temporal MMS fields (Torbert et al., 2016) and plasma suites (Pollock et al., 2016) are used to investigate the structure and dynamics of FTEs. The four MMS spacecraft were maintained at a tetrahedron formation at an average separation of $10 \mathrm{~km}$ (Burch et al., 2016).

FTE identification procedure is done via applying the minimum variance analysis (MVA) on magnetic field measurements where the FTE axis lies in the M (intermediate) direction while $\mathrm{N}$ (minimum) and L (maximum) signify the normal and tangential components, respectively (Xiao, 2004). Our event selection criteria only allows FTEs with 1) small impact parameter (IP < 0.5), and 2) cylindrical symmetry (force-free model goodness of fit parameter, $\chi^{2}<0.1$; cf. AkhavanTafti et al., 2018). A complete list of all 63 burst-mode FTEs used in this study and the force-free flux rope model results are provided in the Supporting Information (Table S1).

Taylor expansion of parameters is enabled by the MMS's multi-dimensional measurements and is used here to compute the barycenteric value:

$$
\mathbf{V}=\mathbf{V}_{\alpha}+\left(\nabla \mathbf{V}_{\alpha}\right) \cdot\left(\overline{\mathbf{r}}-\mathbf{r}_{\alpha}\right)
$$

where $\alpha$ indicates the satellite's number $(\alpha=1, \ldots, 4), \overline{\mathbf{r}}$ denotes the barycentric position and is the average of all 4 spacecraft position vectors, $\mathbf{r}_{\alpha}, \mathbf{V}$ is an $n$-dimensional vector (scalar: $n=1$ and 3D vector: $n=3$ ), and:

$$
(\nabla \mathrm{V})_{\mathrm{ij}}=\frac{1}{\mathrm{~N}^{2}}\left[\sum_{\alpha, \beta \neq \alpha}\left(\mathrm{V}_{\alpha \mathrm{i}}-\mathrm{V}_{\beta \mathrm{i}}\right)\left(\mathrm{r}_{\alpha \mathrm{k}}-\mathrm{r}_{\beta \mathrm{k}}\right)\right] \mathrm{R}_{\mathrm{kj}}^{-1} .
$$

where the symbol $\Sigma_{\alpha \neq \beta}$ indicates summation over all $\mathrm{N}(\mathrm{N}-1) / 2$ independent terms with $\alpha \neq \beta$. Here, $r$ and $\mathrm{N}$ signify the position and the number of spacecraft, respectively, and $\mathrm{R}_{\mathrm{kj}}^{-1}$ is the inverse of the volumetric tensor:

$$
\mathrm{R}_{\mathrm{kj}}=\frac{1}{\mathrm{~N}} \sum_{\alpha=1}^{\mathrm{N}} \mathrm{r}_{\alpha \mathrm{k}} \mathrm{r}_{\alpha \mathrm{j}}
$$

where $i, j, k=1, \ldots, n$ (Harvey, 1998). Equation 2 is also used to calculate the Lorentz force and electron and ion pressure terms in the momentum equation:

$$
\rho \mathrm{du} / \mathrm{dt}=1 / \mu_{0}(\mathbf{B} \cdot \boldsymbol{\nabla}) \mathbf{B}-\nabla\left(\frac{\mathbf{B}^{2}}{2 \mu_{0}}\right)-\nabla p
$$

where $d \boldsymbol{u} / d t$ on the left-hand side of the equation is a total convective time derivative. The terms on the right-hand side are magnetic curvature force, magnetic pressure gradient, and (electron and ion) thermal pressure gradient forces, respectively. The thermal pressure gradients are calculated assuming an isotropic (scalar) pressure, $p$. 


\subsection{Assumptions}

This study relies on three fundamental assumptions to identify FTEs and to obtain a simple empirical model to investigate FTE structures, dynamics, and evolution. First, FTEs are modeled as force-free flux ropes in order to determine their scale size, impact parameter, and cylindricity (goodness-of-fit; $\chi^{2}<0.1$ ). Second, FTEs are assumed axisymmetric to obtain radial profiles of their magnetic and plasma characteristics. Third, FTE diameter is used as a proxy to determine their stage of evolution. To accurately study FTE evolution, individual FTEs should be tracked and studied as a function of time and/or distance from their place of origin. However, this is unattainable with present in-situ observational capabilities (i.e., small number of closely-spaced satellites). To address this, Akhavan-Tafti et al. (2018) used FTE diameter as a proxy to determine FTEs' stage of evolution and, therefore, to study how different physical parameters change. The present study will further investigate how FTE characteristics and dynamics evolve as a function of FTE diameter.

In this study, it is further assumed that the internal properties remain relatively constant along the axis of the FTEs. To test this assumption, the 55 subsolar quasi-force-free FTEs are divided into two categories. The first category (35 events) includes only the FTEs that are observed inside the magnetosheath and are labelled as MSH. The second category (20 events) involves FTEs that are observed at or within $<10$ seconds from a magnetopause crossing and are labelled as MP. Table I summarizes the average thermal, $P_{t h}$, and magnetic, $P_{m}$, pressures inside and outside each FTE category. The ratio of average pressures is also determined (ratio $=P_{\text {inside }} /$ $P_{\text {outside }}$ ); where ratio $>1$ indicates that the average pressure inside is larger than the average pressure outside the FTEs. It is shown that thermal pressure averages are nearly similar (i.e., $P_{t h}$, inside $=P_{\text {th }}$, outside $)$ inside and outside both MSH and MP FTE categories. However, the average magnetic pressure is found to be larger inside both FTE categories (i.e., $P_{m \text {, inside }}>P_{m \text {, outside) }}$. More importantly, it is revealed that the internal magnetic and thermal properties of an FTE do not necessarily change depending on where along the FTEs' axes observations are made. This suggests that FTEs are closed structures and, therefore, their internal properties remain relatively constant along the axis of the structure making them independent of the spacecraft location. We take advantage of this conclusion to study the properties and the evolution of the 55 FTEs irrespective of where along the axis of each FTE, in the magnetosheath or the magnetosphere, the spacecraft traversed.

\section{Analysis and Results}

\subsection{FTE Physical Properties}

Assuming cylindrical symmetry, the constant- $\alpha$ force-free flux rope model is used to infer the size, the core magnetic field strength, the magnetic flux content, and the spacecraft 
trajectories through the FTEs. Having the knowledge of the trajectory of the spacecraft through the FTE enables statistical study of the structure of quasi-force-free flux ropes as a function of distance from the central axis (Burlaga, 1988; Lepping et al., 1990; Slavin et al., 2003). The model defines impact parameter (IP) as the spacecraft distance from the axis of the flux rope at the closest approach (see Figure 11 in Akhavan-Tafti et al. (2018)). Figure 2-4 provide single and multi-spacecraft cross-sectional views of the magnetic and plasma characteristics of the 55 FTEs. IP $=0$ indicates the central region of the FTE and $\mathrm{IP}=1$ denotes the FTE 'edge', beyond which the magnetic field connectivity changes and magnetic fields are no longer bound to the structure (Rijnbeek et al., 1987; Farrugia et al., 1988, 2016). The assumption here is that variables inside the FTE are axisymmetric.

Physical variables are binned (bin width; $\mathrm{BW}=0.1$ ) and averaged across all 55 FTEs. Environmental variability such as location can change average parameters inside FTEs. In order to take these variabilities into account, every data point within each FTE was normalized with an assigned 'weight' (i.e., the ratio of FTE-average of a variable to the average of the variable between all 55 FTEs). The detailed procedure is summarized in Appendix A. The error bars indicate the normalized variations of parameters inside individual bins and is known as the standard error, $\sigma_{\text {mean }} \equiv \sigma / \sqrt{n}$; where $\sigma$ and $n$ are the standard deviation and the number of events in each bin, respectively. Furthermore, the contribution from the FPI instrumental measurement errors are negligible compared to the reported plasma moments as suggested by the crosscomparison of magnetic field and plasma moments at the spacecraft barycenter (cf. Figure 9 in Akhavan-Tafti et al., 2018).

Figure 2a-c shows how plasma moments vary across the FTEs. As shown in panel a, plasma density is lower closer to the central region of the flux rope. The plasma density dip suggests that flux ropes are open-ended (three-dimensional) allowing plasma to evacuate upon strengthening magnetic field due to the twisting of the field lines (Ma et al., 1994; Zhang et al., 2010; Øieroset et al., 2016; Akhavan-Tafti et al., 2018). The dashed line indicates that the average plasma density inside FTEs at the subsolar magnetopause to be $\langle\bar{N}\rangle \pm \sigma_{\bar{N}}=28 \pm 3 \mathrm{~cm}^{-3}$. Panel $b$ indicates that electron and ion velocities, $\left\langle\bar{V}_{e}\right\rangle=190 \mathrm{~km} / \mathrm{s}$ and $\left\langle\bar{V}_{i}\right\rangle=230 \mathrm{~km} / \mathrm{s}$, respectively, are independent of the distance from the FTE's central axis. Electron and ion temperatures vary similarly as a function of IP. Panel $c$ shows that the temperatures are lowest at the outer edge of the FTE. Electron and ion temperatures reach their maxima at $\mathrm{IP}=0.5$ before dropping to their averages, $\left\langle\bar{T}_{e}\right\rangle=45 \mathrm{eV}$ and $\left\langle\bar{T}_{i}\right\rangle=420 \mathrm{eV}$, respectively, near the central axis.

Panel $d$ in Figure 2 shows the twisting of the field lines inside an FTE. The magnetic field twist, $\theta_{B}$, is a function of the ratio of the tangential, $B_{L}$, to the axial, $B_{M}$, components of magnetic field inside the FTE, $\theta_{B}=\operatorname{atan}\left(B_{L} / B_{M}\right)$. In particular, it indicates that the axial component of the field lines becomes dominant near the central axis. The tangential component of the magnetic field becomes larger near the edges. 
Panel $e$ demonstrates the cross-sectional variability of both magnetic, $P_{m}$, and thermal, $P_{t h}$, pressures inside quasi-force-free subsolar FTEs. Magnetic pressure, $\left\langle\bar{P}_{m}\right\rangle=0.65 \mathrm{nPa}$, intensifies near the central axis where total magnetic field peaks. In contrast, thermal pressure, $\left\langle\bar{P}_{t h}\right\rangle=1.6 \mathrm{nPa}$, reaches a minimum near the central axis corresponding to the dip in plasma density. The thermal pressure profile suggests that plasma density variations, not temperature, is the dominant factor determining the plasma pressure profile inside FTEs. More importantly, the pressures are shown to be inversely correlated. A first-order approximation of the pressure gradients inside FTEs (i.e., $\nabla P \sim \Delta P / R_{F R}$, where $R_{F R}$ is the FTE radius) also suggests that the magnetic and thermal pressure gradients point in opposite directions and nearly balance each other.

Panel $f$ further shows that plasma beta, which is the ratio of thermal and magnetic pressures, $\beta=P_{t h} / P_{m}$, dips below its average value of $\langle\bar{\beta}\rangle=4.3$ near the FTE's central axis. The average plasma beta is larger than those reported inside typical FTEs. This is consistent with AkhavanTafti et al. (2018) where newly-born FTEs were shown to contain large plasma density. Over time, plasma will be depleted while magnetic field enhances inside the FTE resulting in lower plasma beta.

During (continuous and secondary) reconnection additional magnetic flux and plasma are added to the outer layers of FTEs. Internal magnetic and thermal forces aim to release this stress and bring the structure back to equilibrium. We first investigate the 'frozen-in' condition (i.e., $\left.\mathbf{E} \sim-\mathbf{v}_{\mathrm{i}} \times \mathbf{B}\right)$ for ions inside the 55 FTEs. The $\mathrm{Y}_{\mathrm{GSE}}$ components of both the electric field measurements (from the Electric Dual Probes (EDP) instrument (Ergun et al., 2016; Lindqvist et al., 2016; Torbert et al., 2016)) and the cross product of ion bulk velocity (from the fast plasma investigation (FPI) instrument (Pollock et al., 2016)) and magnetic field measurements (from the fluxgate magnetometer (FGM) instrument (Russell et al., 2016)) are binned into bins (BW=0.5 $[\mathrm{mV} / \mathrm{m}])$ and are shown in Figure $3 a$. The dashed black line represents a line of slope unity for reference $(\mathrm{y}=\mathrm{x})$. The solid red line represents the fit $(\mathrm{y}=1.01 \mathrm{x}-0.31)$ determined using orthogonal linear regression indicating that ions remain frozen-in inside our quasi-force-free FTEs.

Second, the average net force components are investigated across our quasi-force-free FTEs. Here, net force refers to the vector sum of the magnetic, $J \times B$, and thermal pressure, $\nabla P$, forces. Figure $3 b$ shows the bin-averaged $(\mathrm{BW}=0.1)$ values of the radial component of the net force on

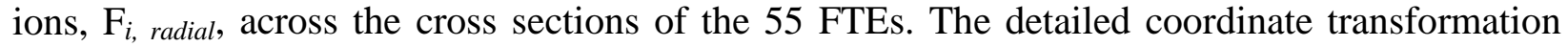
procedure is summarized in Appendix $\mathrm{B}$. Here, a negative value of $\mathrm{F}_{i \text {, radial }}$ indicates inward force. We find that the net force is radially inward. Farther away from the FTE axis, the magnitude of the radially-inward net force enhances. The bin-averaged values of the axial component of the net force, $\mathrm{F}_{i \text {, axial }}$, across the cross section of the FTEs are further shown in Figure $\mathbf{3} c$. In contrast with the radial component, the axial component of the net force is found to enhance significantly near the FTE's central axis, IP < 0.5. In conclusion, the results found in Figure 3a-c suggest that the 'frozen-in' magnetic field lines are compressed inward with the plasma giving rise to 
strengthening core magnetic field. However, due to the strengthening magnetic field the radiallyinward net force is reduced near the FTE's central axis while the axial net force enhances. These findings indicate that plasmas are accelerated toward the FTE's central axis, though closer to the FTE's core region the radial force is reduced and the plasma is instead accelerated along the axial direction causing the plasma to escape the stressed region (e.g., Ma et al., 1994).

Figure 4 further provides the cross sectional profiles of the radial components of the magnetic and thermal forces across the FTE. The error bars indicate the standard error inside each individual bin. The radial profiles indicate that inside FTEs magnetic pressure gradient (blue shade) and ion thermal pressure gradient (black shade) are dominant near the FTE's central axis. It is also found that magnetic curvature force (red shade) is radially inward and becomes more significant near the FTE's edge. As expected, electron thermal pressure gradient (green shade) is small throughout the structure. On the other hand, ion thermal pressure gradient remains large, relatively steady, and radially inward across the structure.

Figure 5 $a$ - $d$ compare the spatial profiles of perpendicular and parallel components of plasma current density, net force, and electron and ion temperatures. In panel $a$, the ratio of perpendicular to parallel components of the current density is shown to be smaller than unity (i.e., $R_{J}=J_{\perp} / J_{\|}<1$; Akhavan-Tafti et al., 2018). This is a result of our event selection criteria where only quasi-force-free FTE candidates were chosen. Panel $b$ shows that the majority of the net force inside the FTE accelerates plasmas perpendicular to the local magnetic field. The relatively large parallel force is consistent with the observed enhanced axial component of the net force inside FTEs. The parallel net force can facilitate the escape of the stressed plasma through accelerating them along the magnetic field. The parallel net force may also affect the helical magnetic structure of the FTEs (Zhao et al., 2016). Temperature anisotropy inside FTEs is evident in panels $c$ - $d$. Parallel electron temperature, $T_{e \|}$, and perpendicular ion temperature, $T_{i \perp}$, are observed to be the larger components of the electron and ion temperatures, respectively.

\subsection{FTE Boundaries}

In this section, the physical characteristics and dynamics are studied inside and just outside the 55 quasi-force-free FTEs. As shown in Figure 6, the term 'inside' refers to the cross section of the flux rope (shaded red) that meets the constant-alpha force-free flux rope model criteria Burlaga, 1988; Lepping et al., 1990; Akhavan-Tafti et al., 2018, while 'outside,' shaded as light blue, indicates the outer perimeter of the flux rope (i.e., the draping region Rijnbeek et al., 1987; Farrugia et al., 1988). The thickness of the outer perimeter was selected at 10 local ion-inertial lengths, $d_{i}$. This thickness is nearly one-third of the nominal FTE diameter at the dayside magnetopause (Akhavan-Tafti et al., 2018) and is sufficiently large to include reconnectionassociated kinetic and MHD effects (Smith, 1977; Tóth et al., 2017).

Table II lists the relative contribution, i.e., percentage of total, of the force terms. The force 
terms are averaged inside and outside the 55 FTEs. The shading in each row corresponds to the relative contribution of individual force terms with the darkest shading representing the largest contribution. The table indicates that magnetic pressure gradient and ion thermal pressure gradient are the dominant force terms contributing to the total force inside and outside FTEs. Magnetic forces (magnetic curvature force and magnetic pressure gradient) are comparable in magnitude to the ion thermal pressure gradient inside FTEs. The thermal pressure gradient becomes dominant outside the FTEs.

Figure 7 compares plasma beta, current density, and net force vector components inside and outside FTEs. Observations outside the 55 FTEs are bin-averaged $\left(\mathrm{BW}=1 d_{i}\right.$; where $d_{i}$ $=c / \omega_{p i}=2.28 \times 10^{7}\left(N_{i}\right)^{-1 / 2} \mathrm{~cm}$, is the average local ion inertial length and is a function of average ion density, $N_{i}$, outside each FTE). Panel a indicates that plasma beta varies quite remarkably across the FTE boundary. This is partly due to the enhanced magnetic pressure inside FTEs. Panel $b$ shows that current density is, on average, enhanced by nearly $50 \%$ inside FTEs. Panels $c$-e present the net force vector components in the cylindrical coordinate system. The radial component of the vector sum of the magnetic and thermal forces, remains relatively small outside the FTEs suggesting that the radial magnetic and thermal forces are balanced. Furthermore, the magnitude of the tangential component of the net force, defined as

$\mathrm{F}_{\text {tangential }}=\left|\mathbf{F}-\left(\mathrm{F}_{\text {radial }} \hat{\mathbf{r}}+\mathrm{F}_{\text {axial }} \widehat{\mathbf{M}}\right)\right|$, enhances near the FTE edge. Finally, the axial component of the net force remains steady and non-zero outside the FTE. The residual tangential and axial net force components may impact the twist of the field lines at the outer layers of the FTEs (e.g., Zhao et al., 2016).

\subsection{Case Study}

The magnetic and thermal force magnitudes inside the 55 quasi-force-free FTEs are nonzero (i.e., $\boldsymbol{J} \times \boldsymbol{B} \neq \mathbf{0} \& \boldsymbol{\nabla P} \neq \mathbf{0}$ ). Here, we provide two quasi-force free FTEs observed in the magnetosheath with enhanced net force components. Figure 8 summarizes the magnetic and plasma moment observations. The panels in the left column include the measurements by MMS2: $a$ ) total magnetic field, $b$ ) magnetic field vector components in the GSE coordinate system, $c$ ) plasma density, $d$ ) ion velocity, $e$ ) plasma current density, and $f$ ) ion temperature. The magnetic and thermal force components are further included in the right column: $g$ ) total magnetic field, $h$ ) magnetic and thermal pressure components, $i$ ) magnetic curvature force, $j$ ) magnetic pressure gradient force, $k$ ) ion thermal pressure gradient force, and $l$ ) net force which is defined as the vector sum of the magnetic and thermal forces. The vector components are presented with respect to the local magnetic field. The component of each vector parallel to B is shown in red, while the magnitude of the perpendicular component of the vector is shown in black. The red gradient and the light-blue solid color shadings represent the areas 'inside' and 'outside' each FTE. The width of the blue shaded area is 10 ion inertial lengths which is determined using the average local ion density, $\mathrm{N}_{\mathrm{i}}=40 \mathrm{~cm}^{-3}$. 
Zhou et al. (2017) reported the presence of an ion jet reversal ( $v_{i z, G S M}= \pm 200 \mathrm{~km} / \mathrm{s}$; panel c) during a magnetopause crossing (November 17, 2015 — 02:13:40 - 02:14:40 UT) corresponding to a 'dissipative' interaction of two neighboring FTEs. Shortly after this crossing, at the peak of the southward ion velocity, a chain of small-scale FTEs is observed, labeled as 'FTE 1' and 'FTE 2'. In particular, two FTEs centered at 02:20:48 UT and 02:21:24 UT are observed surrounded by two southward $v_{i z}$ peaks (02:17:30 UT and 02:22 UT) which met our selection criteria (Akhavan-Tafti et al., 2018).

Plasma density is shown to dip by $50 \%$ at the center of FTE 1 and by $25 \%$ inside FTE 2. Parallel current density is further found to enhance inside the two events. Ion temperature anisotropy is observed across both FTEs; however, the parallel ion temperature, $\mathrm{T}_{\mathrm{i} / /}$, is smaller

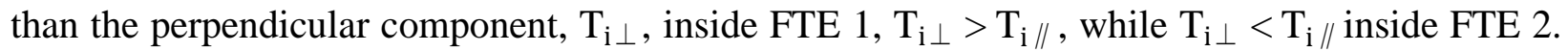
Magnetic pressure enhances inside both FTEs resulting in increased magnetic pressure gradient force. $\beta=P_{t h} / P_{m} \sim 4$ outside the two FTEs. Plasma beta is reduced inside both structures. Ion thermal pressure gradient force is found dominant inside the two FTEs.

\section{Discussion}

In this study, we investigate the structural characteristics of the 55 subsolar quasi-force-free FTEs identified and modeled by Akhavan-Tafti et al. (2018). The determination of the impact parameter of the MMS spacecraft trajectories through modeling FTEs as force-free flux ropes with cylindrical geometry allows the study of cross-sectional profiles of physical parameters as a function of distance from the FTEs' central axis. It is revealed that:

i) Plasma density and plasma beta increase with increasing distance from the FTE's central axis.

ii) The radial component of the net force (solid-black arrow in Figure 9a) at the outer layers of the flux rope points inward. The axial component of the force, shown as an into-the-plane arrow, becomes significant near the FTE's central axis.

As illustrated in Figure 9a, the continuous supply of magnetic flux and plasma to the FTEs' outer layers, shown as grey-shaded area, by magnetic reconnection at adjacent $\mathrm{X}$-lines and/or coalescence with the neighboring FTEs, shown as X points, drives FTEs to 'grow' (e.g., Hasegawa et al., 2010; Akhavan-Tafti et al., 2018) while also contributing to:

$a$. the observed average decrease in plasma density near the FTE's central axis. Crosssectional profile of plasma density is represented as a blue and red-shaded area. As discussed above, plasma density drops below average, white-shaded area, near the FTE's core region.

$b$. The observed radially-inward net force at the outer layers of the FTEs as well as the 
enhanced axial net force closer to the FTE's central axis.

These observations are consistent with the continuous supply of magnetic flux and plasma heating by magnetic reconnection. As suggested by the radially-inward net force, the additional magnetic flux and plasma, in the reconnection exhaust, is compressed and accelerated, respectively, while being transported toward the FTE's core region. Closer to the FTE's central axis, where $\mathrm{B}_{\text {axial }}$ enhances, the axial net force component strengthens. The strengthening axial force can then accelerate plasmas along the FTE's axis and cause the 'trapped' plasma population to escape the stressed region (e.g., Ma et al., 1994; Akhavan-Tafti et al., 2018; Sun et al., 2019).

iii) The tangential component, $B_{L}$, of the magnetic field increases farther away from the FTE's central axis. Figure $9 b$ shows the profile of magnetic pitch angle, $\theta_{B}=\operatorname{atan}\left({ }^{B} /_{B_{M}}\right)$, across the cross section of a typical quasi-force-free FTE. The tangential component of the magnetic field enhances at the FTE's outer layers. The FTEs' magnetic pitch angle may impact the coalescence process (e.g., Yamada et al., 1990).

iv) Ion and electron temperatures are found to be anisotropic throughout the cross section of FTE-type flux ropes observed both inside the magnetosheath and at the magnetopause.

a. We observe that, on average, $\mathrm{T}_{e} / / \mathrm{T}_{e \perp}$ throughout the cross section of subsolar FTEs. Firehose, $T_{e \|}>T_{e \perp}$, and whistler, $\mathrm{T}_{e \perp}>\mathrm{T}_{e / l}$, instabilities can arise due to electron temperature anisotropy (e.g., Kennel \& Petschek, 1966; Hollweg \& Völk, 1970a, 1970b; Pilipp \& Völk, 1971; Cuperman, 1981; Gary \& Madland, 1985; Zhao et al., 1996; Drake et al., 2010; Wang et al., 2019). Zhao et al. (1996) notes that the former condition is rarely observed in space plasmas and, hence, infrequently reported in the literature, but our analysis suggests that it may occur in FTEs.

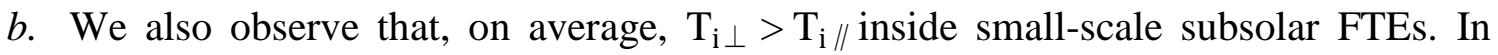
theory, anisotropic ion temperature, $\mathrm{T}_{\mathrm{i} \perp}>\mathrm{T}_{\mathrm{i} / /}$, can act as a source of free energy for various low-frequency electromagnetic instabilities in the magnetosheath, such as the ion cyclotron anisotropy and instability and the mirror instability (e.g., Gary et al., 1976; Lee et al., 1988; Midgley \& Davis, 1963; Remya et al., 2013; Zwan \& Wolf, 1976). A future MMS study will examine anisotropies in the plasma moment distributions and the associated instabilities inside FTEs.

The profiles of the magnetic and plasma properties are further analyzed outside FTEs to gain insight into the role of environmental conditions in impacting the evolution, e.g., growth, of FTEs. In particular, it is shown that, compared to the average properties inside FTEs:

i) Plasma beta enhances significantly, by a factor of $>4$, outside FTEs. This may be the result of enhanced thermal pressure, and reduced magnetic pressure, at the adjacent X- 
lines.

ii) Current density is reduced outside FTEs.

iii) The radial component of the net force remains steady and negligible outside FTEs which may be a result of our selection process, i.e., quasi-force-free FTEs. However, the tangential and the axial components of the net force are found to be significant outside FTEs. These observations suggest that the structure of FTEs, in particular, the magnetic field twist at the FTE's outer layers, $\theta_{B}=\operatorname{atan}\left(B_{L} / B_{M}\right)$, may be affected by environmental net forces (e.g., Zhao et al., 2016).

The observed internal and external magnetic and plasma characteristics suggest that FTEs evolve with time. Previously, the FTEs at the subsolar magnetopause have been found to range between $10^{2}-10^{4} \mathrm{~km}$ in diameter. Akhavan-Tafti et al. (2018) suggested that FTEs are generated at ion-scale at the subsolar magnetopause and grow as they convect away toward flanks and high-latitude magnetopause where the magnetosheath pressure is much lower (Sibeck et al., 1990; Eastwood et al., 2012). From the three FTE growth mechanisms discussed in Figure 1, a radially-outward net force across the FTE boundary is required to cause the 'expansion' of FTEs as a function of distance from the subsolar region. However, our observations indicate that the net force inside FTEs is radially inward and that the radial component of the net force is negligible, i.e., force balance $\boldsymbol{J} \times \boldsymbol{B}-\boldsymbol{\nabla P} \sim 0$, outside FTEs. To further confirm or deny 'expansion' as an FTE growth mechanism, we examine the plasma pressure inside FTEs as a function of FTE diameter.

In the absence of magnetic reconnection, the irreversible adiabatic expansion of FTEs requires, cf. Appendix $\mathrm{C}$, that the thermal pressure inside an ion-scale FTE to drop by at least two orders of magnitude, i.e., $p<10^{-4} \mathrm{nPa}$, while the FTE grows macro-scale, $>1 \mathrm{R}_{\mathrm{E}}$ in diameter. Figure 10 $a$ shows histograms of thermal $\left(P_{t h}=N k_{B} T\right.$; where $T=T_{i}+T_{e}$ and $k_{B}$ is the Boltzmann constant) and magnetic pressures measured inside the 55 FTEs at the subsolar magnetopause. The thermal and magnetic pressure distributions are nearly symmetric about their means at 1.5 $\mathrm{nPa}\left(0.3<P_{t h}[\mathrm{nPa}]<5.0\right)$ and $0.5 \mathrm{nPa}\left(0.03<P_{m}[\mathrm{nPa}]<3\right)$, respectively. Therefore, our observations do not confirm FTE growth via expansion.

We further examine the rate at which pressure varies as a function of FTE size. Figure 10 $b$ shows the change in average thermal, $P_{t h}$, and magnetic pressures, $P_{m}$, as a function of FTE diameter. Here, pressures are averaged across the cross section of individual FTEs and plotted with respect to the FTE diameter. The 55 FTEs are grouped into bins (bin widths $(\mathrm{BW})=$ $500 \mathrm{~km})$ and the error bars indicate standard error of values inside each bin $\left(\sigma_{\text {mean }} \equiv \sigma / \sqrt{n}\right.$; where $n$ is the number of events in each bin). The solid curves represent power law fits to the data with shaded areas signifying the $95 \%$ confidence interval. The dependence of thermal pressure as a function of the FTE diameter, $\lambda$, is measured on average as $\lambda^{-0.24}$ compared to $\lambda^{-3.3}$, 
shown as a grey dashed-line, expected for adiabatic expansion of FTEs. This observed slow decay of the thermal pressure is consistent with plasma undergoing significant heating (Drake et al., 2006) further indicating that adiabatic expansion alone cannot explain the observed FTE range of diameters at the magnetopause. This result suggests that adiabatic FTE expansion in the subsolar magnetosheath is accompanied by processes that add $i$ ) magnetic flux, and ii) thermal pressure to the outer layers of FTEs. Hence, FTE growth can occur via three mechanisms:

1) Continuous reconnection: Ongoing multiple $\mathrm{X}$-line reconnection can add magnetic layers to an existing FTE as predicted by simulations (Lee \& Fu, 1985; Chen et al., 2017). The resulting increase in the structures' magnetic flux and plasma content can explain also the exponential distribution of magnetic flux inside subsolar FTEs (Fermo et al., 2011; Akhavan-Tafti et al., 2018). Further, plasma is heated in the exhausts of the primary X-line and plasma pressure is expected to increase in the outer layers of these flux ropes as new layers of helical magnetic flux are added and the FTE grows in size.

2) Coalescence: PIC simulations suggest that numerous small-scale flux ropes form in thin current sheets (e.g., Drake \& Lee, 1977a, 1977b; Galeev et al., 1978; Hesse et al., 1999; Pritchett, 2001, 2005; Ricci et al., 2004; W. Daughton et al., 2011; Nakamura et al., 2016; Huang et al., 2016; Lapenta et al., 2018). Neighboring small-scale flux ropes then reconnect with each other and, as a result, grow into larger merged FTEs (e.g., Wang et al., 2016; Alm et al., 2017; Zhou et al., 2017). Through coalescence, the magnetic flux content of the merged FTE is equal to or greater than the larger of the two (i.e., Fermo et al., 2011). However, plasma is added to the outer layers of the merged FTE and heating may occur as a result of the reconnection between the outer layers of the two merging FTEs.

3) Adiabatic expansion due to the pressure-imbalance across the FTE boundary: Upon formation at the subsolar magnetopause, FTEs advect with the magnetosheath flow toward the flanks and high-latitude regions where the magnetosheath thermal pressure is lower. The pressure-imbalance across the FTE boundary created as they are carried tailward may lead to the expansion of FTEs (Eastwood et al., 2012). The expansion will continue until the internal magnetic forces and plasma pressure gradients are again in balance. FTEs expand to relieve the stress most likely produced by reconnection-driven heating of their outer layers.

\section{Conclusions}

In summary, we have used the high temporal and spatial-resolution MMS magnetic and plasma measurements to investigate the structure and dynamics of 55 subsolar FTEs. The forcefree flux rope model was initially used to derive the relative distance of the spacecraft with respect to the FTE's central axis. i.e., the impact parameter. Having knowledge of the impact parameter facilitates the statistical study of cross-sectional profiles of physical parameters inside 
and outside FTEs. In particular, we observe that plasma density and plasma beta decrease near the FTE's central axis. We also find that the sum of the magnetic and thermal forces points radially inward at the outer layers of FTEs suggesting that plasmas and the frozen-in magnetic flux are accelerated toward the center of the FTE causing the field lines to compress. However, closer to the FTE's central axis, the axial net force is found to dominate and to accelerate the plasma along the FTE's axis and out of the region of compressing field lines.

Magnetic and plasma characteristics are also studied outside FTEs to gain insight into how changing external conditions may impact FTEs. It is observed that plasma beta outside the FTEs is, on average, significantly larger than the values inside. Similarly, the tangential and axial net force components are also found to enhance outside FTEs. Since magnetic field pitch angle is a function of the ratio of the tangential and axial magnetic field components, the enhanced tangential and axial net force components can likely impact the twist of magnetic field lines at the FTEs' outer layers.

We also find that the average thermal pressure inside FTEs decreases with increasing FTE diameter. If the 55 FTEs had started out with similar diameters and external conditions, the observed rate of thermal pressure decrease with increasing FTE diameter is shown to be slower than the rate expected for the adiabatic expansion of FTEs. This result suggests that the adiabatic expansion of FTEs at the subsolar magnetosheath is accompanied by processes, such as magnetic reconnection, that add $i$ ) magnetic flux, and $i$ ) thermal pressure to the outer layers of FTEs. Magnetic reconnection at adjacent X-lines and/or coalescence with the neighboring FTEs is most likely the external source of magnetic flux and plasma. This continuous addition of magnetic flux and plasma, while causing an FTE to grow in size, drives a net radially-inward force which leads to the compression of the magnetic field lines and the acceleration of plasmas inside the FTE. This process leads naturally to the low-beta core region of the FTE as it evolves toward its final relaxed, force-free state, i.e., the Taylor state (Taylor, 1986).

\section{Acknowledgements}

The authors would like to thank the MMS team for providing high-quality data products and analysis tools. The data can be publicly accessed at the MMS Science Data Center website (https://lasp.colorado.edu/mms/sdc/public/). The helpful discussions with G. Tòth and W. Sun of the University of Michigan are greatly appreciated. The work done at the University of Michigan was supported by NASA MMS contract NNG04EB99C at Southwest Research Institute. JPE is supported by UKRI Science and Technology Facilities Council (STFC) grant ST/N000692/1.

\section{Appendix A}

An analytical weight determines that the $i_{t h}$ observation comes from a sub-population with a particular mean and variance, $\sigma^{2} / w_{i}$, where $\sigma^{2}$ is a common variance and $w_{i}$ is the weight of the $i_{t h}$ 
observation. Analytical weights are commonly used in meta-analyses where each observation is the mean of a sample (Kish, 1992). In this study, each observed data point, $X_{i, n}$, is weighted with:

$$
w_{n}=\langle X\rangle / \bar{X}_{n}
$$

where $w_{n}$ is the analytical weight assigned to data points inside the $n_{t h}$ FTE. Here, $\langle X\rangle \equiv \frac{1}{P} \sum_{n=1}^{P} \bar{X}_{n}$, where $P=55$ and is the total number of FTEs used in this study. $\bar{X}_{n}$ indicates the average value of variable $X$ across the cross section of the $n_{t h}$ FTE, hence it is defined as:

$$
\bar{X}_{n} \equiv \frac{1}{N} \sum_{i=1}^{N} X_{i, n},
$$

where subscript $i=1, \ldots, N$ denotes individual data points inside an FTE with total $N$ measurements.

\section{Appendix B}

In order to determine the radial component of a vector in a force-free flux rope, the vector is projected from the LMN coordinates into the cylindrical coordinates. Figure $\mathbf{1 1}$ demonstrates that the vector, $\boldsymbol{A}$, is first projected into the flux rope cross section plane (LN-plane). The vector can then be written in terms of the in-plane components, $\boldsymbol{A}(\widehat{\boldsymbol{N}}, \widehat{\boldsymbol{L}})=A_{N} \widehat{\boldsymbol{N}}+A_{L} \widehat{\boldsymbol{L}}$; where unit vector $\widehat{\boldsymbol{N}}$ points along the spacecraft trajectory and $\widehat{\boldsymbol{L}}=\widehat{\boldsymbol{M}} \times \widehat{\boldsymbol{N}}$. The transformation into the cylindrical coordinates requires the knowledge of angle $\boldsymbol{\theta}$ which is a function of 1) the distance of the spacecraft location along its trajectory $(0<X<d$; where $d$ indicates the distance between the two flux rope edges) from the flux rope's central axis, and 2$)$ the closest approach $\left(d_{\text {closest }}\right.$ approach $=r_{\text {flux } \text { rope }} \times$ IP):

$$
\theta=\tan ^{-1}\left(\frac{\left(\frac{X-d / 2}{r_{\text {flux rope }}}\right)}{\boldsymbol{I P}}\right)
$$

where $r_{\text {flux rope }}$ is the flux rope radius as determined by the force-free flux rope model. The radial component of the vector can be written as:

$$
A_{r}=A_{N} \sin (\theta)
$$

It is important to note that for statistical analyses, all flux ropes should be rotated into the same LMN coordinates. To achieve this, all flux ropes are rotated with respect to the $\widehat{N}$ axis for their axes, $\widehat{\boldsymbol{M}}$, to point in the same (e.g., dawn-dusk) direction. Because we are only interested in the radial component of the vector the different flux rope helicities (e.g., right-hand helicity) will not 
impact the results.

\section{Appendix C}

The second law of thermodynamics states that the entropy inside an irreversibly expanding FTE must not decrease:

$$
p_{2} V_{2}^{\gamma} \geq p_{1} V_{1}^{\gamma}
$$

where pressure $p \propto N T$, where $N$ and $T$ are zeroth (density) and second (temperature) plasma moments, respectively. $V$ denotes the flux rope volume, hence, proportional to the square of the FTE diameter $\left(V \propto \lambda^{2} H\right.$; where $\lambda$ is the FTE diameter and $\mathrm{H}$ is the FTE length, assuming cylindrical symmetry) (e.g., Lingam \& Comisso, 2018). The length, H, of the FTE is assumed to remain constant. It is important to note that recent observational evidence suggest that reconnection sites can expand, though primarily in local time along the boundary, which may result in a change in $\mathrm{H}$ (Zou et al., 2018). The subscripts indicate the stage of evolution ( $\mathrm{t}_{1}$ and $\mathrm{t}_{2} \gg \mathrm{t}_{1}$ ) and $\gamma=5 / 3$ is the adiabatic specific heat ratio for monoatomic gases. Thereby, the equation above can be written as:

$$
p_{2} / p_{1} \geq\left(\lambda_{2} / \lambda_{1}\right)^{-3.3}
$$

where $\lambda$ is the FTE diameter.

The role of magnetic field in the above approximation can also be included by using the double-adiabatic approximation (e.g., Baumjohann \& Treumann, 2012). This approximation takes into account the different variations in the parallel and perpendicular pressures in the presence of a sufficiently strong magnetic field. The perpendicular thermal pressure $\mathrm{P}_{\perp}$ is a linear function of plasma density $n$ and magnetic field magnitude $B$ as suggested by the conservation of magnetic moment, $\mu=\mathrm{P}_{\perp} / \mathrm{nB}$, in systems wherein plasma gyration takes longer than temporal variations of the plasma. In the absence of any dissipative mechanisms and heat transfer, the parallel component of thermal pressure is also found to be a function of both plasma density and magnetic field:

$$
\begin{gathered}
\mathrm{P}_{\perp} \propto \mathrm{nB} \\
\mathrm{P}_{\|} \propto \mathrm{n}^{3} \mathrm{~B}^{-2}
\end{gathered}
$$

It is further shown that the parallel $\gamma_{\|}$and the perpendicular $\gamma_{\perp}$ adiabatic indices are defined in the double-adiabatic equations of state as:

$$
\gamma_{\perp}=1+\frac{\ln \left(\mathrm{B}_{2} / \mathrm{B}_{1}\right)}{\ln \left(\mathrm{n}_{2} / \mathrm{n}_{1}\right)}, \text { and }
$$




$$
\gamma_{\|}=3-2 \frac{\ln \left(\mathrm{B}_{2} / \mathrm{B}_{1}\right)}{\ln \left(\mathrm{n}_{2} / \mathrm{n}_{1}\right)}
$$

Therefore, inside an adiabatically growing flux rope, wherein no heat transfer is assumed, the thermal pressure becomes increasingly anisotropic, i.e., $\mathrm{P}_{\perp} / \mathrm{P}_{\|} \neq 1$. Plasma pressure components can then be written as:

$$
\begin{gathered}
p_{\perp 2} / p_{\perp 1} \geq\left(\lambda_{2} / \lambda_{1}\right)^{-2 \gamma_{\perp}}, \text { and } \\
p_{\| 2 / p_{\| 1}} \geq\left(\lambda_{2} / \lambda_{1}\right)^{-2 \gamma_{\|}}
\end{gathered}
$$

Given the MMS observations, Akhavan-Tafti et al. [2018] found that plasma density of subsolar FTEs is, to a first-order approximation, a linear function of the flux rope core magnetic field, $\mathrm{n}$ $\left[\mathrm{cm}^{-3}\right]=-0.36|\mathrm{~B}|[\mathrm{nT}]+43.81$. Here, we find that:

$$
\begin{gathered}
p_{\perp 2 / p_{\perp 1}} \geq\left(\lambda_{2} / \lambda_{1}\right)^{-5.9}, \text { and } \\
p_{\| 2 / p_{\| 1}} \geq\left(\lambda_{2} / \lambda_{1}\right)^{+1.9},
\end{gathered}
$$

which suggests that for small-scale FTEs to grow macro-scale via adiabatic expansion their internal pressure must become dominantly parallel. This is not physically possible since the strong anisotropy will trigger plasma instabilities, e.g., firehose instability, to return the plasma population to a lower energy state. Dissipative mechanisms and/or plasma energization mechanisms including magnetic reconnection can further thermalize plasma and prevent anisotropy.

This article is protected by copyright. All rights reserved. 


\section{References}

Akhavan-Tafti, M., Slavin, J. A., Le, G., Eastwood, J. P., Strangeway, R. J., Russell, C. T., ... Akhavan-Tafti, M. (2018). MMS Examination of FTEs at the Earth's Subsolar Magnetopause. Journal of Geophysical Research: Space Physics. https://doi.org/10.1002/2017JA024681

Alm, L., Farrugia, C. J., Paulson, K. W., Argall, M. R., Torbert, R. B., Burch, J. L., ... Giles, B. L. (2017). Differing properties of two ion-scale magnetopause flux ropes. Journal of Geophysical Research: Space Physics. https://doi.org/10.1002/2017JA024525

Baalrud, S. D., Bhattacharjee, A., \& Huang, Y.-M. (2012). Reduced magnetohydrodynamic theory of oblique plasmoid instabilities. Physics of Plasmas, 19(2), 22101.

Baumjohann, W., \& Treumann, R. A. (2012). Basic space plasma physics. World Scientific Publishing Company.

Berchem, J., \& Russell, C. T. (1984). Flux transfer events on the magnetopause: Spatial distribution and controlling factors. Journal of Geophysical Research: Space Physics, 89(A8), 6689-6703.

Bhattacharjee, A., Huang, Y.-M., Yang, H., \& Rogers, B. (2009). Fast reconnection in highLundquist-number plasmas due to the plasmoid instability. Physics of Plasmas, 16(11), 112102.

Biskamp, D. (1982). Effect of secondary tearing instability on the coalescence of magnetic islands. Physics Letters A, 87(7), 357-360.

Biskamp, Dieter. (1986). Magnetic reconnection via current sheets. The Physics of Fluids, 29(5), $1520-1531$.

Biskamp, Dieter. (1996). Magnetic reconnection in plasmas. Astrophysics and Space Science, 242(1-2), 165-207.

Burch, J. L., Moore, T. E., Torbert, R. B., \& Giles, B. L. (2016). Magnetospheric multiscale overview and science objectives. Space Science Reviews, 199(1-4), 5-21.

Burlaga, L. F. (1988). Magnetic clouds and force-free fields with constant alpha. Journal of Geophysical Research, 93(7), 7217. https://doi.org/10.1029/JA093iA07p07217

Cassak, P. A., Shay, M. A., \& Drake, J. F. (2009). Scaling of Sweet--Parker reconnection with secondary islands. Physics of Plasmas, 16(12), 120702.

Chen, L.-J., Bessho, N., Lefebvre, B., Vaith, H., Fazakerley, A., Bhattacharjee, A., ... others. (2008). Evidence of an extended electron current sheet and its neighboring magnetic island during magnetotail reconnection. Journal of Geophysical Research: Space Physics, 113(A12). 
Chen, Y., Tóth, G., Cassak, P., Jia, X., Gombosi, T. I., Slavin, J. A., ... Henderson, M. G. (2017). Global Three-Dimensional Simulation of Earth's Dayside Reconnection Using a Two-Way Coupled Magnetohydrodynamics With Embedded Particle-in-Cell Model: Initial Results. Journal of Geophysical Research: Space Physics, 122(10), 10,318-10,335. https://doi.org/10.1002/2017JA024186

Comisso, L., Grasso, D., \& Waelbroeck, F. L. (2015). Phase diagrams of forced magnetic reconnection in Taylor's model. Journal of Plasma Physics, 81(5).

Comisso, L, Lingam, M., Huang, Y.-M., \& Bhattacharjee, A. (2016). General theory of the plasmoid instability. Physics of Plasmas, 23(10), 100702.

Comisso, Luca, \& Grasso, D. (2016). Visco-resistive plasmoid instability. Physics of Plasmas, 23(3), 32111.

Cuperman, S. (1981). Electromagnetic kinetic instabilities in multicomponent space plasmas: Theoretical predictions and computer simulation experiments. Reviews of Geophysics, 19(2), 307-343.

Daughton, W., Roytershteyn, V., Albright, B. J., Karimabadi, H., Yin, L., \& Bowers, K. J. (2009). Transition from collisional to kinetic regimes in large-scale reconnection layers. Physical Review Letters, 103(6), 065004. https://doi.org/10.1103/PhysRevLett.103.065004

Daughton, W., Roytershteyn, V., Karimabadi, H., Yin, L., Albright, B. J., Bergen, B., \& Bowers, K. J. (2011). Role of electron physics in the development of turbulent magnetic reconnection in collisionless plasmas. Nature Physics, 7(7), 539-542. https://doi.org/10.1038/nphys 1965

Daughton, W, Roytershteyn, V., Karimabadi, H., Yin, L., Albright, B. J., Gary, S. P., \& Bowers, K. J. (2011). Secondary island formation in collisional and collisionless kinetic simulations of magnetic reconnection. In AIP Conference Proceedings (Vol. 1320, pp. 144-159).

Daughton, William, Scudder, J., \& Karimabadi, H. (2006). Fully kinetic simulations of undriven magnetic reconnection with open boundary conditions. Physics of Plasmas, 13(7), 72101.

Deng, X. H., Matsumoto, H., Kojima, H., Mukai, T., Anderson, R. R., Baumjohann, W., \& Nakamura, R. (2004). Geotail encounter with reconnection diffusion region in the Earth's magnetotail: Evidence of multiple X lines collisionless reconnection? Journal of Geophysical Research: Space Physics, 109(A5).

Ding, D. Q., Lee, L. C., \& Swift, D. W. (1992). Particle simulations of driven collisionless magnetic reconnection at the dayside magnetopause. Journal of Geophysical Research: Space Physics, 97(A6), 8453-8481.

Dong, X.-C., Dunlop, M. W., Trattner, K. J., Phan, T. D., Fu, H.-S., Cao, J.-B., ... others. (2017). Structure and evolution of flux transfer events near dayside magnetic reconnection dissipation region: MMS observations. Geophysical Research Letters. 
Dorelli, J. C., \& Bhattacharjee, A. (2009). On the generation and topology of flux transfer events. Journal of Geophysical Research: Space Physics, 114(6), 1-21.

https://doi.org/10.1029/2008JA013410

Drake, J. F., Opher, M., Swisdak, M., \& Chamoun, J. N. (2010). A magnetic reconnection mechanism for the generation of anomalous cosmic rays. The Astrophysical Journal, 709(2), 963-974. https://doi.org/10.1088/0004-637X/709/2/963

Drake, J. F., \& Lee, Y. C. (1977a). Kinetic theory of tearing instabilities. The Physics of Fluids, 20(8), 1341-1353.

Drake, J. F., \& Lee, Y. C. (1977b). Nonlinear evolution of collisionless and semicollisional tearing modes. Physical Review Letters, 39(8), 453.

Drake, J. F., Swisdak, M., Che, H., \& Shay, M. A. (2006). Electron acceleration from contracting magnetic islands during reconnection. Nature, 443(7111), 553.

https://doi.org/10.1038/nature05116

Eastwood, J. P., Phan, T. D., Mozer, F. S., Shay, M. A., Fujimoto, M., Retin??, A., ... Dandouras, I. (2007). Multi-point observations of the Hall electromagnetic field and secondary island formation during magnetic reconnection. Journal of Geophysical Research: Space Physics, 112(6), 1-13. https://doi.org/10.1029/2006JA012158

Eastwood, J. P., Phan, T. D., Fear, R. C., Sibeck, D. G., Angelopoulos, V., Ieroset, M., ... Shay, M. A. (2012). Survival of flux transfer event (FTE) flux ropes far along the tail magnetopause. Journal of Geophysical Research: Space Physics, 117(8), 1-9. https://doi.org/10.1029/2012JA017722

Ergun, R. E., Tucker, S., Westfall, J., Goodrich, K. A., Malaspina, D. M., Summers, D., ... Cully, C. M. (2016). The axial double probe and fields signal processing for the MMS mission. Space Science Reviews, 199(1-4), 167-188. https://doi.org/10.1007/s11214-0140115-x

Farrugia, C. J., Lavraud, B., Torbert, R. B., Argall, M., Kacem, I., Yu, W., ... Strangeway, R. J. (2016). MMS Observations and Non-Force Free Modeling of a Flux Transfer Event Immersed in a Super-Alfvénic Flow. Geophysical Research Letters, 43(12), 1-8. https://doi.org/10.1002/2016GL068758

Farrugia, C. J., Rijnbeek, R. P., Saunders, M. A., Southwood, D. J., Rodgers, D. J., Smith, M. F., ... Woolliscroft, L. J. C. (1988). A multi-instrument study of flux transfer event structure. Journal of Geophysical Research, 93(A12), 14465.

https://doi.org/10.1029/JA093iA12p14465

Fermo, R. L., Drake, J. F., Swisdak, M., \& Hwang, K. J. (2011). Comparison of a statistical model for magnetic islands in large current layers with Hall MHD simulations and Cluster FTE observations. Journal of Geophysical Research: Space Physics, 116(A9), n/a-n/a. 
https://doi.org/10.1029/2010ja016271

Finn, J. M., \& Kaw, P. K. (1977). Coalescence instability of magnetic islands. The Physics of Fluids, 20(1), 72-78.

Furno, I., Intrator, T. P., Hemsing, E. W., Hsu, S. C., Abbate, S., Ricci, P., \& Lapenta, G. (2005). Coalescence of two magnetic flux ropes via collisional magnetic reconnection. Physics of Plasmas, 12(5), 55702.

Galeev, A. A., Coroniti, F. V, \& Ashour-Abdalla, M. (1978). Explosive tearing mode reconnection in the magnetospheric tail. Geophysical Research Letters, 5(8), 707-710.

Gary, S. P., Montgomery, M. D., Feldman, W. C., \& Forslund, D. W. (1976). Proton temperature anisotropy instabilities in the solar wind. Journal of Geophysical Research, 81(7), 12411246. https://doi.org/10.1029/JA081i007p01241

Gary, S. P., \& Madland, C. D. (1985). Electromagnetic electron temperature anisotropy instabilities. Journal of Geophysical Research: Space Physics, 90(A8), 7607-7610.

Gonzalez, W., \& Parker, E. (2016). Magnetic Reconnection: Concepts and Applications (Vol. 427). Springer.

Harvey, C. C. (1998). Spatial Gradients and the Volumetric Tensor. Retrieved from http://www.issibern.ch/forads/sr-001-12.pdf

Hasegawa, H., Wang, J., Dunlop, M. W., Pu, Z. Y., Zhang, Q.-H. Q.-H. H., Lavraud, B., ... Bogdanova, Y. V. (2010). Evidence for a flux transfer event generated by multiple X-line reconnection at the magnetopause. Geophysical Research Letters, 37(16), 1-6. https://doi.org/10.1029/2010GL044219

Hesse, M., Schindler, K., Birn, J., \& Kuznetsova, M. (1999). The diffusion region in collisionless magnetic reconnection. Physics of Plasmas, 6(5), 1781-1795.

Hoilijoki, S, Ganse, U., Sibeck, D. G., Cassak, P. A., Turc, L., Battarbee, M., .. others. (2019). Properties of magnetic reconnection and FTEs on the dayside magnetopause with and without positive IMF B component during southward IMF. Journal of Geophysical Research: Space Physics.

Hoilijoki, Sanni, Ganse, U., Pfau-Kempf, Y., Cassak, P. A., Walsh, B. M., Hietala, H., ... Palmroth, M. (2017). Reconnection rates and X line motion at the magnetopause: Global 2D-3V hybrid-Vlasov simulation results. Journal of Geophysical Research: Space Physics, 122(3), 2877-2888.

Hollweg, J. V., \& Völk, H. J. (1970a). Two New Plasma Instabilities in the Solar Wind. Nature, 225(5231), 441-443. https://doi.org/10.1038/225441a0

Hollweg, J. V, \& Völk, H. J. (1970b). New plasma instabilities in the solar wind. Journal of Geophysical Research, 75(28), 5297-5309.

This article is protected by copyright. All rights reserved. 
Huang, S. Y., Sahraoui, F., Retino, A., Le Contel, O., Yuan, Z. G., Chasapis, A., .. Burch, J. L. (2016). MMS observations of ion-scale magnetic island in the magnetosheath turbulent plasma. Geophysical Research Letters, 43(15), 7850-7858.

https://doi.org/10.1002/2016GL070033

Huang, Y.-M., \& Bhattacharjee, A. (2010). Scaling laws of resistive magnetohydrodynamic reconnection in the high-Lundquist-number, plasmoid-unstable regime. Physics of Plasmas, 17(6), 62104. https://doi.org/10.1063/1.3420208

Huang, Y.-M., \& Bhattacharjee, A. (2013). Plasmoid instability in high-Lundquist-number magnetic reconnection a. Physics of Plasmas, 20(5), 55702.

Huang, Y.-M., Bhattacharjee, A., \& Sullivan, B. P. (2011). Onset of fast reconnection in Hall magnetohydrodynamics mediated by the plasmoid instability. Physics of Plasmas, 18(7), 72109.

Hwang, K-J, Sibeck, D. G., Burch, J. L., Choi, E., Fear, R. C., Lavraud, B., ... others. (2018). Small-scale flux transfer events formed in the reconnection exhaust region between two Xlines. Journal of Geophysical Research: Space Physics.

Hwang, K.-J., Sibeck, D. G., Giles, B. L., Pollock, C. J., Gershman, D., Avanov, L., ... Burch, J. L. (2016). The substructure of a flux transfer event observed by the MMS spacecraft. Geophysical Research Letters, 43(18), 9434-9443. https://doi.org/10.1002/2016GL070934

Karimabadi, H., Dorelli, J., Roytershteyn, V., Daughton, W., \& Chacón, L. (2011). Flux Pileup in Collisionless Magnetic Reconnection: Bursty Interaction of Large Flux Ropes. Physical Review Letters, 107(2), 025002. https://doi.org/10.1103/PhysRevLett.107.025002

Kennel, C. F., \& Petschek, H. E. (1966). Limit on stably trapped particle fluxes. Journal of Geophysical Research, 71(1), 1-28.

Kish, L. (1992). Weighting for unequal Pi. Journal of Official Statistics, 8(2), 183.

Kumar, A., \& Rust, D. M. (1996). Interplanetary magnetic clouds, helicity conservation, and current-core flux-ropes. Journal of Geophysical Research: Space Physics, 101(A7), 1566715684.

Lapenta, G., Pucci, F., Olshevsky, V., Servidio, S., Sorriso-Valvo, L., Newman, D. L., \& Goldman, M. V. (2018). Nonlinear waves and instabilities leading to secondary reconnection in reconnection outflows. Journal of Plasma Physics, 84(1).

Lee, L. C., \& Fu, Z. F. (1985). A theory of magnetic flux transfer at the Earth's magnetopause. Geophysical Research Letters, 12(2), 105-108. https://doi.org/10.1029/GL012i002p00105

Lee, L. C., \& Fu, Z. F. (1986). Multiple X line reconnection: 1. A criterion for the transition from a single $\mathrm{X}$ line to a multiple $\mathrm{X}$ line reconnection. Journal of Geophysical Research: Space Physics, 91(A6), 6807-6815.

This article is protected by copyright. All rights reserved. 
Lee, L. C., Price, C. P., Wu, C. S., \& Mandt, M. E. (1988). A study of mirror waves generated downstream of a quasi-perpendicular shock. Journal of Geophysical Research: Space Physics, 93(A1), 247-250.

Lepping, R. P., Jones, J. A., \& Burlaga, L. F. (1990). Magnetic field structure of interplanetary magnetic clouds at 1 AU. Journal of Geophysical Research, 95(A8), 11957. https://doi.org/10.1029/JA095iA08p11957

Lindqvist, P.-A. P.-A., Olsson, G., Torbert, R. B., King, B., Granoff, M., Rau, D., ... others. (2016). The spin-plane double probe electric field instrument for MMS. Space Science Reviews, 199(1-4), 137-165. https://doi.org/10.1007/s11214-014-0116-9

Lingam, M., \& Comisso, L. (2018). A maximum entropy principle for inferring the distribution of 3D plasmoids. Physics of Plasmas, 25(1), 12114.

Loureiro, N. F., Schekochihin, A. A., \& Cowley, S. C. (2007). Instability of current sheets and formation of plasmoid chains. Physics of Plasmas, 14(10), 100703. https://doi.org/10.1063/1.2783986

Loureiro, N. F., Schekochihin, A. A., \& Uzdensky, D. A. (2013). Plasmoid and KelvinHelmholtz instabilities in Sweet-Parker current sheets. Physical Review E, 87(1), 13102.

Loureiro, N. F., Samtaney, R., Schekochihin, A. A., \& Uzdensky, D. A. (2012). Magnetic reconnection and stochastic plasmoid chains in high-Lundquist-number plasmas. Physics of Plasmas, 19(4), 42303.

Ma, Z. W. T., Otto, A., \& Lee, L. C. (1994). Core magnetic field enhancement in single X line, multiple X line and patchy reconnection. Journal of Geophysical Research: Space Physics, 99(A4), 6125-6136.

Midgley, J. E., \& Davis, L. (1963). Calculation by a moment technique of the perturbation of the geomagnetic field by the solar wind. Journal of Geophysical Research, 68(18), 5111-5123.

Milan, S. E., Lester, M., Cowley, S. W. H., \& Brittnacher, M. (2000). Convection and auroral response to a southward turning of the IMF: Polar UVI, CUTLASS, and IMAGE signatures of transient magnetic flux transfer at the magnetopause. J. Geophys. Res., 105(A7), 1574115755. https://doi.org/10.1029/2000JA900022

Murphy, N. A., Young, A. K., Shen, C., Lin, J., \& Ni, L. (2013). The plasmoid instability during asymmetric inflow magnetic reconnection. Physics of Plasmas, 20(6), 61211.

Nakamura, T. K. M., Nakamura, R., Narita, Y., Baumjohann, W., \& Daughton, W. (2016). Multi-scale structures of turbulent magnetic reconnection. Physics of Plasmas, 23(5), 52116.

Ni, L., Kliem, B., Lin, J., \& Wu, N. (2015). Fast magnetic reconnection in the solar chromosphere mediated by the plasmoid instability. The Astrophysical Journal, 799(1), 79.

This article is protected by copyright. All rights reserved. 
Øieroset, M., Phan, T. D., Haggerty, C., Shay, M. A., Eastwood, J. P., Gershman, D. J., ... Malakit, K. (2016). MMS observations of large guide field symmetric reconnection between colliding reconnection jets at the center of a magnetic flux rope at the magnetopause. Geophysical Research Letters, 43(11), 5536-5544. https://doi.org/10.1002/2016GL069166

Omidi, N., Blanco-Cano, X., Russell, C. T., \& Karimabadi, H. (2006). Global hybrid simulations of solar wind interaction with Mercury: Magnetosperic boundaries. Advances in Space Research, 38(4), 632-638.

Omidi, N., \& Sibeck, D. G. (2007). Flux transfer events in the cusp. Geophysical Research Letters, 34(4), L04106. https://doi.org/10.1029/2006GL028698

Phan, T. D., Paschmann, G., Twitty, C., Mozer, F. S., Gosling, J. T., Eastwood, J. P., ... Lucek, E. A. (2007). Evidence for magnetic reconnection initiated in the magnetosheath. Geophysical Research Letters, 34(14).

Pilipp, W., \& Völk, H. J. (1971). Analysis of electromagnetic instabilities parallel to the magnetic field. Journal of Plasma Physics, 6(1), 1-17.

Pollock, C., Moore, T., Jacques, A., Burch, J., Gliese, U., Saito, Y., ... Zeuch, M. (2016). Fast Plasma Investigation for Magnetospheric Multiscale. Space Science Reviews, 199(1-4), 331-406. https://doi.org/10.1007/s11214-016-0245-4

Pritchett, P. L. (1992). The coalescence instability in collisionless plasmas. Physics of Fluids B: Plasma Physics, 4(10), 3371-3381.

Pritchett, P. L. (2001). Collisionless magnetic reconnection in a three-dimensional open system. Journal of Geophysical Research: Space Physics, 106(A11), 25961-25977.

Pritchett, P. L. (2005). Onset and saturation of guide-field magnetic reconnection. Physics of Plasmas, 12(6), 62301.

Pritchett, P. L., \& Coroniti, F. V. (2004). Three-dimensional collisionless magnetic reconnection in the presence of a guide field. Journal of Geophysical Research: Space Physics, 109(A1).

Pritchett, P. L., \& Wu, C. C. (1979). Coalescence of magnetic islands. The Physics of Fluids, 22(11), 2140-2146.

Raeder, J. (2006). Flux transfer events: 1. Generation mechanism for strong southward IMF. In Annales Geophysicae (Vol. 24, pp. 381-392).

Remya, B., Reddy, R. V., Tsurutani, B. T., Lakhina, G. S., \& Echer, E. (2013). Ion temperature anisotropy instabilities in planetary magnetosheaths. Journal of Geophysical Research: Space Physics, 118(2), 785-793. https://doi.org/10.1002/jgra.50091

Retinò, A., Nakamura, R., Vaivads, A., Khotyaintsev, Y., Hayakawa, T., Tanaka, K., ... others. (2008). Cluster observations of energetic electrons and electromagnetic fields within a reconnecting thin current sheet in the Earth's magnetotail. Journal of Geophysical

This article is protected by copyright. All rights reserved. 
Research: Space Physics, 113(A12).

Ricci, P., Brackbill, J. U., Daughton, W., \& Lapenta, G. (2004). Collisionless magnetic reconnection in the presence of a guide field. Physics of Plasmas, 11(8), 4102-4114.

Rijnbeek, R. P., Cowley, S. W. H., Southwood, D. J., \& Russell, C. T. (1984). A survey of dayside flux transfer events observed by ISEE 1 and 2 magnetometers. Journal of Geophysical Research: Space Physics, 89(A2), 786-800.

Rijnbeek, R. P., Farrugia, C. J., Southwood, D. J., Dunlop, M. W., Mier-Jedrzejowicz, W. A. C., Chaloner, C. P., ... Smith, M. F. (1987). A magnetic boundary signature within flux transfer events. Planetary and Space Science, 35(7), 871-878. https://doi.org/10.1016/00320633(87)90065-1

Russell, C. T., Anderson, B. J., Baumjohann, W., Bromund, K. R., Dearborn, D., Fischer, D., ... Richter, I. (2016). The Magnetospheric Multiscale Magnetometers. Space Science Reviews, 199(1-4), 189-256. https://doi.org/10.1007/s11214-014-0057-3

Russell, Christopher T, \& Elphic, R. C. (1978). Initial ISEE magnetometer results: Magnetopause observations. Space Science Reviews, 22(6), 681-715.

Scholer, M. (1988). Magnetic flux transfer at the magnetopause based on single X line bursty reconnection. Geophysical Research Letters, 15(4), 291-294.

Sibeck, D. G., Lepping, R. P., \& Lazarus, A. J. (1990). Magnetic Field Line Draping in the Plasma Depletion Layer. JOURNAL OF GEOPHYSICAL RESEARCH, 95(1), 2433-2440. https://doi.org/10.1029/JA095iA03p02433

Slavin, J. A., Lepping, R. P., Gjerloev, J., Fairfield, D. H., Hesse, M., Owen, C. J., .. Mukai, T. (2003). Geotail observations of magnetic flux ropes in the plasma sheet. Journal of Geophysical Research, 108(A1), 1015. https://doi.org/10.1029/2002JA009557

Slavin, James A, Imber, S. M., Boardsen, S. A., DiBraccio, G. A., Sundberg, T., Sarantos, M., ... others. (2012). MESSENGER observations of a flux-transfer-event shower at Mercury. Journal of Geophysical Research: Space Physics, 117(A12).

Smith, D. F. (1977). Current instability in reconnecting current sheets. Journal of Geophysical Research, 82(4), 704-708.

Song, H.-Q., Chen, Y., Li, G., Kong, X.-L., Feng, S.-W., \& others. (2012). Coalescence of macroscopic magnetic islands and electron acceleration from STEREO observation. Physical Review X, 2(2), 21015. https://doi.org/10.1103/PhysRevX.2.021015

Stanier, A., Daughton, W., Simakov, A. N., Chacón, L., Le, A., Karimabadi, H., ... Bhattacharjee, A. (2017). The role of guide field in magnetic reconnection driven by island coalescence. Physics of Plasmas, 24(2), 022124. https://doi.org/10.1063/1.4976712

Sun, W. J., Slavin, J. A., Tian, A. M., Bai, S. C., Poh, G. K., Akhavan-Tafti, M., ... others. 
(2019). MMS study of the structure of ion-scale flux ropes in the Earth's cross-tail current sheet. Geophysical Research Letters.

Taylor, Jb. (1986). Relaxation and magnetic reconnection in plasmas. Reviews of Modern Physics, 58(3), 741.

Teh, W.-L. W.-L., Nakamura, T. K. M., Nakamura, R., Baumjohann, W., Russell, C. T., Pollock, C., ... others. (2017). Evolution of a typical ion-scale magnetic flux rope caused by thermal pressure enhancement. Journal of Geophysical Research: Space Physics, 122(2), 20402050. https://doi.org/10.1002/2016JA023777

Teh, W. -L., Denton, R. E., Sonnerup, B. U. Ö., \& Pollock, C. (2017). MMS observations of oblique small-scale magnetopause flux ropes near the ion diffusion region during weak guide-field reconnection. Geophysical Research Letters, 44(13), 6517-6524. https://doi.org/10.1002/2017GL074291

Tian, H., Yao, S., Zong, Q., He, J., \& Qi, Y. (2010). Signatures of magnetic reconnection at boundaries of interplanetary small-scale magnetic flux ropes. The Astrophysical Journal, $720(1), 454$.

Torbert, R. B., Russell, C. T., Magnes, W., Ergun, R. E., Lindqvist, P.-A., LeContel, O., ... Lappalainen, K. (2016). The FIELDS Instrument Suite on MMS: Scientific Objectives, Measurements, and Data Products. Space Science Reviews, 199(1-4), 105-135. https://doi.org/10.1007/s11214-014-0109-8

Tóth, G., Chen, Y., Gombosi, T. I., Cassak, P., Markidis, S., \& Peng, I. B. (2017). Scaling the ion inertial length and its implications for modeling reconnection in global simulations. Journal of Geophysical Research: Space Physics, 122(10).

Wang, R., Lu, Q., Nakamura, R., Baumjohann, W., Russell, C. T., Burch, J. L., ... others. (2017). Interaction of magnetic flux ropes via magnetic reconnection observed at the magnetopause. Journal of Geophysical Research: Space Physics, 122(10).

Wang, R., Lu, Q., Nakamura, R., Huang, C., Du, A., Guo, F., ... Wang, S. (2016). Coalescence of magnetic flux ropes in the ion diffusion region of magnetic reconnection. Nature Physics, 12(3), 263-267. https://doi.org/10.1038/nphys3578

Wang, S., Wang, R., Yao, S. T., Lu, Q., Russell, C. T., \& Wang, S. (2019). Anisotropic Electron Distributions and Whistler Waves in a Series of the Flux Transfer Events at the Magnetopause. Journal of Geophysical Research: Space Physics, 124(3), 1753-1769.

Xiao, C. J., Pu, Z. Y., Ma, Z. W., Fu, S. Y., Huang, Z. Y., \& Zong, Q. G. (2004). Inferring of flux rope orientation with the minimum variance analysis technique. Journal of Geophysical Research, 109(A11), A11218. https://doi.org/10.1029/2004JA010594

Yamada, M, Ono, Y., Hayakawa, A., Katsurai, M., \& Perkins, F. W. (1990). Magnetic reconnection of plasma toroids with cohelicity and counterhelicity. Physical Review Letters, 
65(6), 721.

Yamada, Masaaki, Kulsrud, R., \& Ji, H. (2010). Magnetic reconnection. REVIEWS OF MODERN PHYSICS, 82(JANUARY-MARCH), 603-664. https://doi.org/10.1103/RevModPhys.82.603

Zhang, H., Kivelson, M. G., Khurana, K. K., McFadden, J., Walker, R. J., Angelopoulos, V., ... others. (2010). Evidence that crater flux transfer events are initial stages of typical flux transfer events. Journal of Geophysical Research: Space Physics, 115(A8). https://doi.org/10.1029/2009JA015013

Zhao, C., Russell, C. T., Strangeway, R. J., Petrinec, S. M., Paterson, W. R., Zhou, M., ... others. (2016). Force balance at the magnetopause determined with MMS: Application to flux transfer events. Geophysical Research Letters, 43(23). https://doi.org/10.1002/2016GL071568

Zhao, J., Sakai, J.-I., \& Nishikawa, K.-I. (1996). Excitation of whistler waves driven by an electron temperature anisotropy. Solar Physics, 168(2), 345-355. https://doi.org/10.1007/BF00148060

Zhou, M., Berchem, J., Walker, R. J. J., El-Alaoui, M., Deng, X., Cazzola, E., ... Burch, J. L. (2017). Coalescence of Macroscopic Flux Ropes at the Subsolar Magnetopause: Magnetospheric Multiscale Observations. Physical Review Letters, 119(5), 055101. https://doi.org/10.1103/PhysRevLett.119.055101

Zhou, Meng, Pang, Y., Deng, X., Huang, S., \& Lai, X. (2014). Plasma physics of magnetic island coalescence during magnetic reconnection. Journal of Geophysical Research: Space Physics, 119(8), 6177-6189. https://doi.org/10.1002/2013JA019483

Zou, Y., Walsh, B. M., Nishimura, Y., Angelopoulos, V., Ruohoniemi, J. M., McWilliams, K. A., \& Nishitani, N. (2018). Spreading Speed of Magnetopause Reconnection X-Lines Using Ground-Satellite Coordination. Geophysical Research Letters, 45(1), 80-89.

Zwan, B. J., \& Wolf, R. A. (1976). Depletion of solar wind plasma near a planetary boundary. Journal of Geophysical Research, 81(10), 1636-1648.

This article is protected by copyright. All rights reserved. 

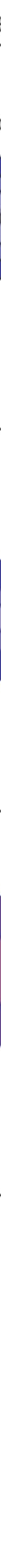

This article is protected by copyright. All rights reserved. 
Figure 1: Three candidate FTE growth mechanisms: a) Adiabatic Expansion, b) Continuous reconnection, and c) coalescence. The concentric circles show magnetic islands which are twodimensional projections of flux ropes. The shading of the islands determine the thermal pressure $p$ inside them with the darkest shades indicating the region of largest $p$. The number of circles per area indicates magnetic field magnitude and the gradient shading inside the islands shows plasma pressure variability across the structure. The number of black circles represents the islands' magnetic flux content $\psi$. The X markers indicate X-points at which magnetic field lines reconnect. The subscripts 1 and 2 indicate earlier and later evolution stages, respectively.

This article is protected by copyright. All rights reserved. 

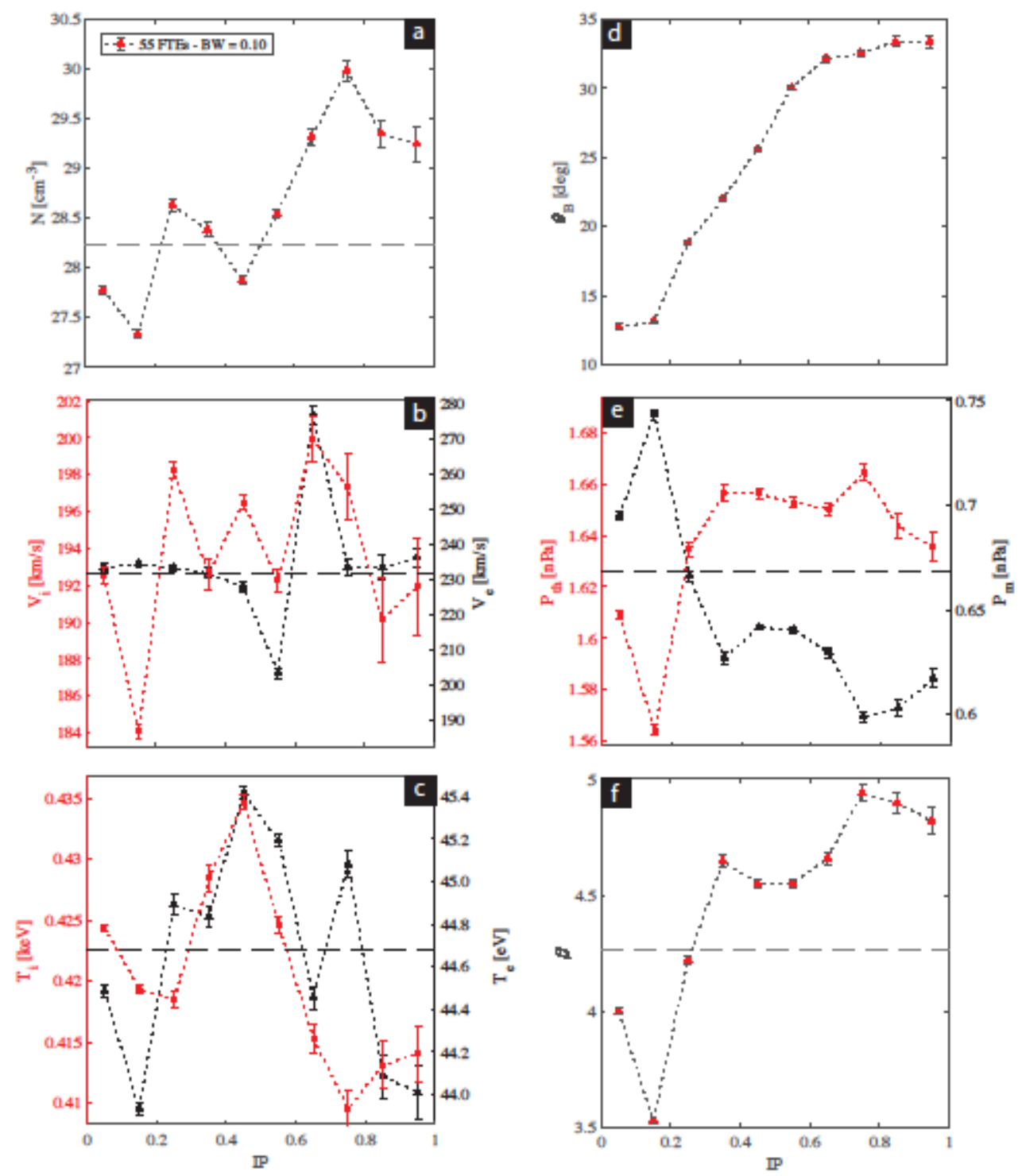
Figure 2: Cross-sectional profiles of a) plasma density, b) ion (left axis; red markers) and electron (right axis; black markers) bulk velocity, $c$ ) ion (left axis; red markers) and electron (right axis; black markers) temperature, d) magnetic pitch angle, $\theta_{B}=$ atan $\left(B_{L} / B_{M}\right)$, where $B_{L}$ and $B_{M}$ represent tangential and axial magnetic field components, respectively, $\left.e\right)$ thermal, $P_{t h}$, (left axis; red markers) and magnetic, $P_{m}$, pressures (right axis; black markers), and f) plasma beta. All data points across the 55 FTEs are grouped and averaged inside bins (bin-width; $B W=0.1)$. The error bar denotes the standard error $\sigma_{\text {mean }}$ and is defined as the normalized variations of a given parameter inside each individual bin, $\sigma_{\text {mean }} \equiv \sigma / \sqrt{n}$; where $\sigma$ and $n$ are the standard deviation and the number of events in each bin, respectively. Note that the primary and secondary y-axes are different in range and, in the case of plasma temperature, unit. 

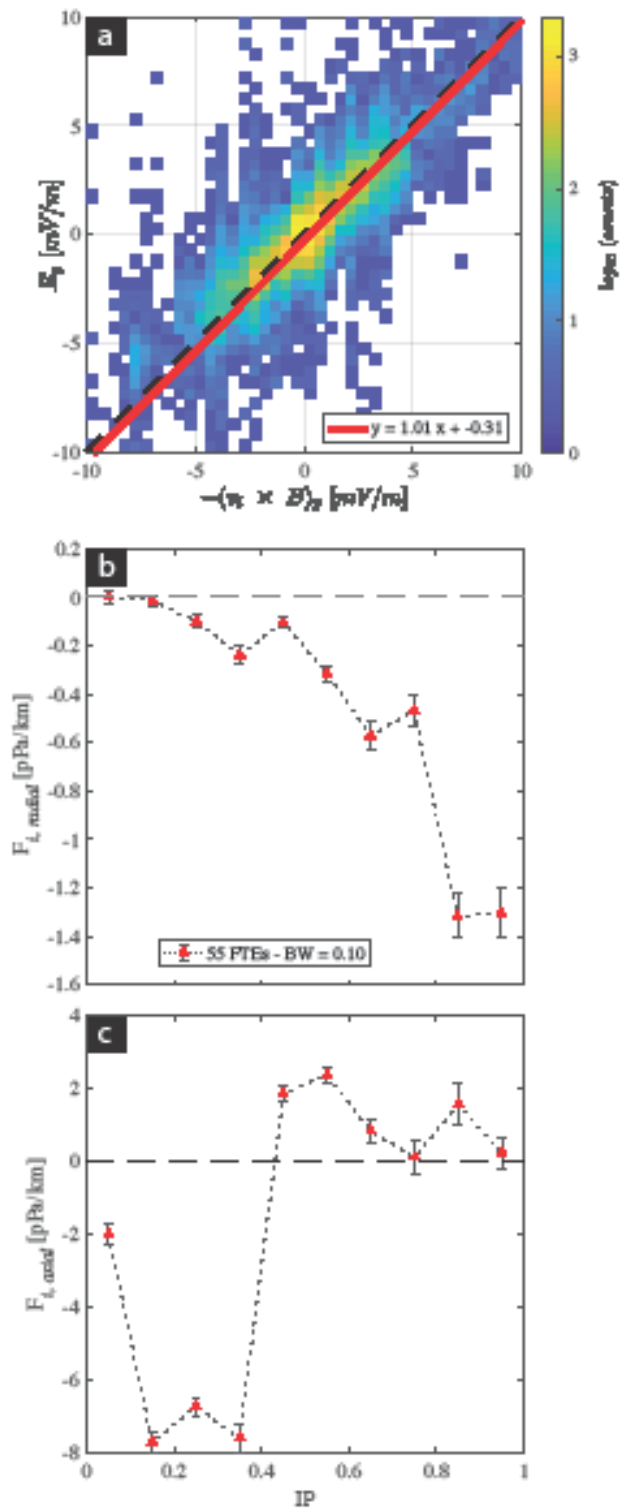
Figure 3: a) Cross-Comparison of the $Y_{G S E}$ components of $-v_{i} x B$ and the electric field measurements grouped and counted inside bins $(B W=0.5[\mathrm{mV} / \mathrm{m}])$. The solid red line represents the orthogonal linear regression fit. The cross sectional profile of the $b$ ) radial component of the net force, and c) axial component of the net force experienced by ions inside the 55 quasi-forcefree FTEs. All data points across the 55 FTEs are grouped and averaged inside bins $(B W=0.1)$. The error bar denotes the standard error inside each individual bin.

This article is protected by copyright. All rights reserved. 


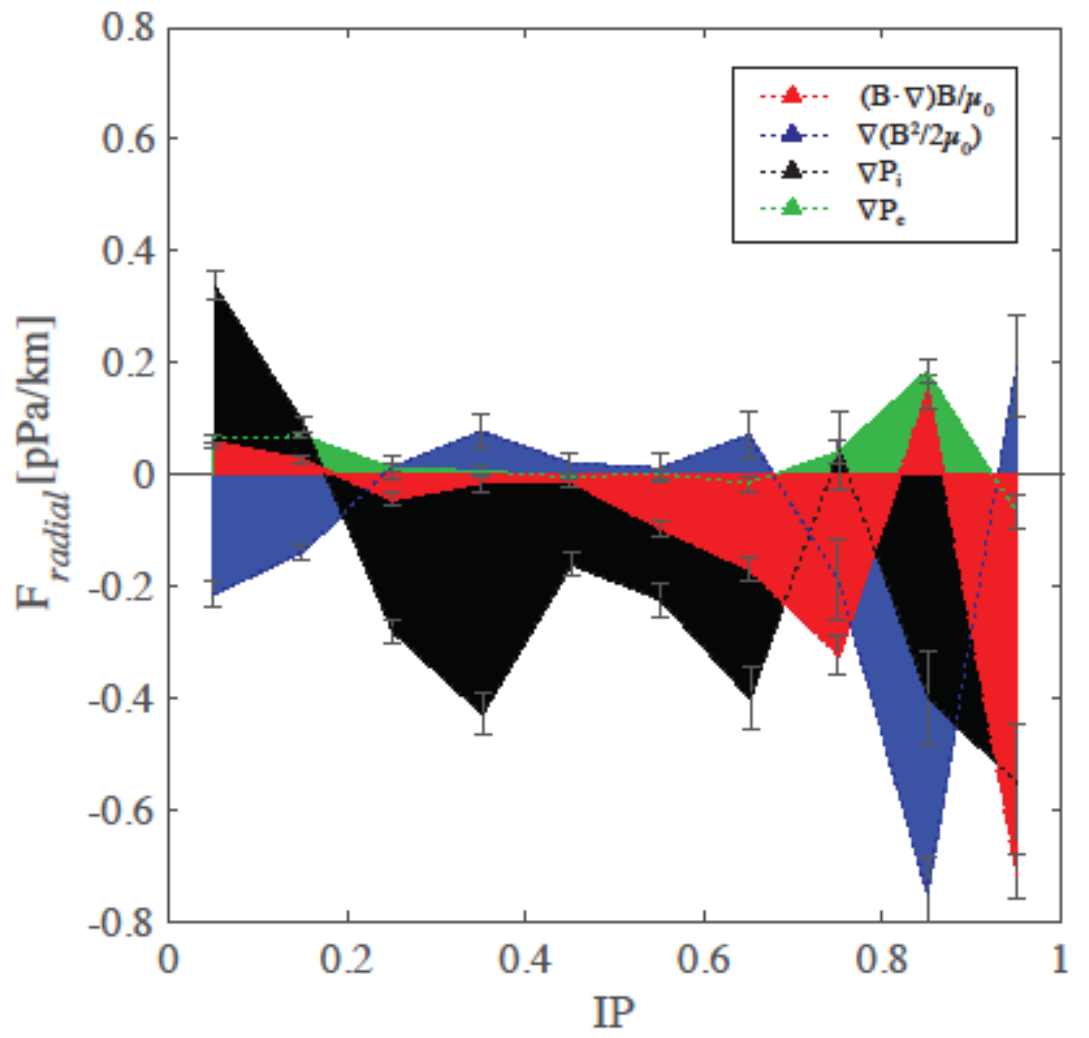

This article is protected by copyright. All rights reserved. 
Figure 4: The radial components of the magnetic and thermal forces as a function of IP. The magnetic forces include the magnetic curvature force (shown in red) and the magnetic pressure gradient (shown in blue). The thermal forces are the ion (black shade) and electron (green shade) thermal pressure gradients forces. All data points across the 55 FTEs are grouped and averaged inside bins $(B W=0.1)$. The error bar denotes the standard error, $\sigma_{\text {mean }}$ and is defined as the normalized variations of a given parameter inside each individual bin, $\sigma_{\text {mean }} \equiv \sigma / \sqrt{n}$; where $\sigma$ and $n$ are the standard deviation and the number of events in each bin, respectively. The dotted lines further show the boundaries of each of the shaded areas. 

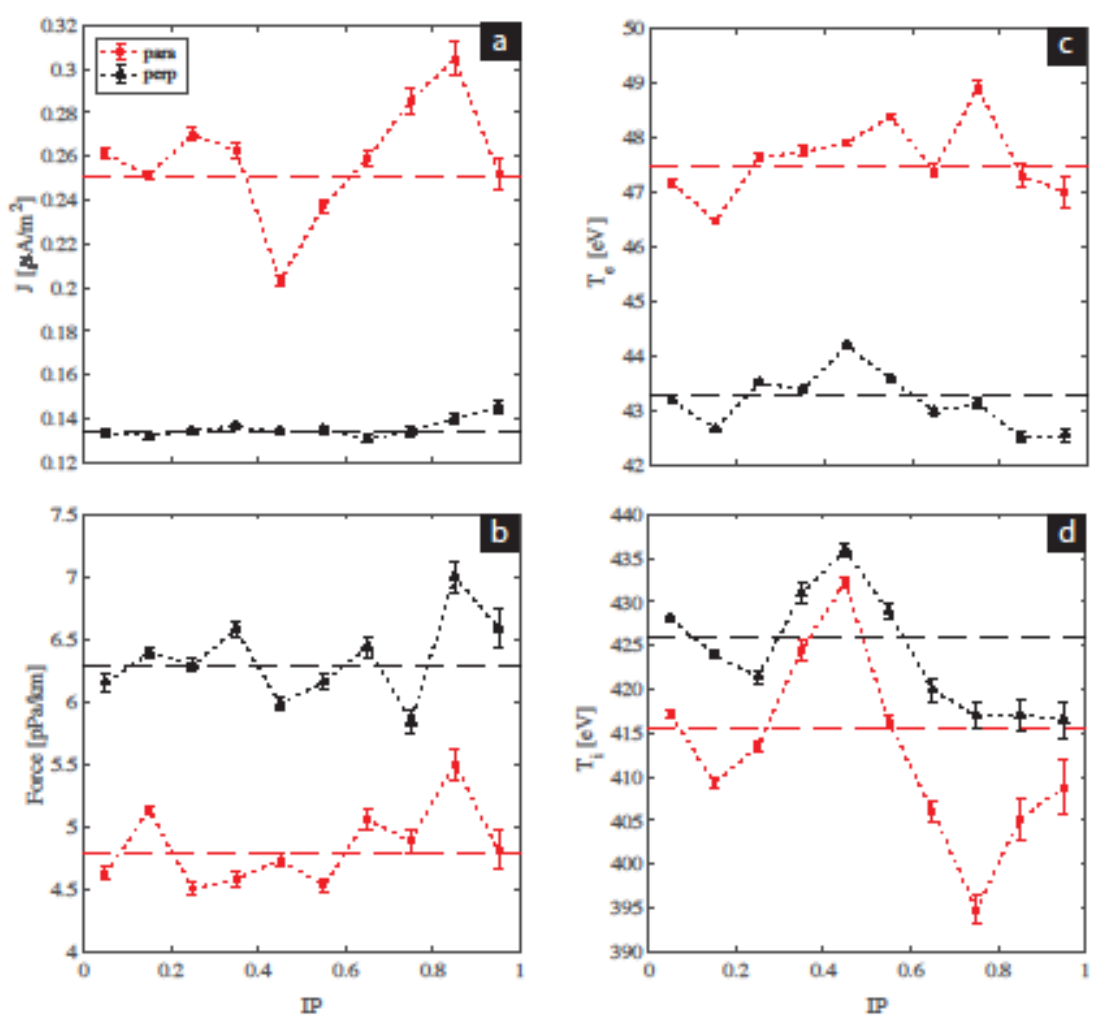
Figure 5: Cross sectional profiles of the parallel (red) and perpendicular (black) components of current density, net force, electron and ion temperatures. All data points across the 55 FTEs are grouped and averaged inside bins $(B W=0.1)$. The error bar denotes the standard error inside each individual bin. The dashed line represents the average value of each parameter across all FTEs. 


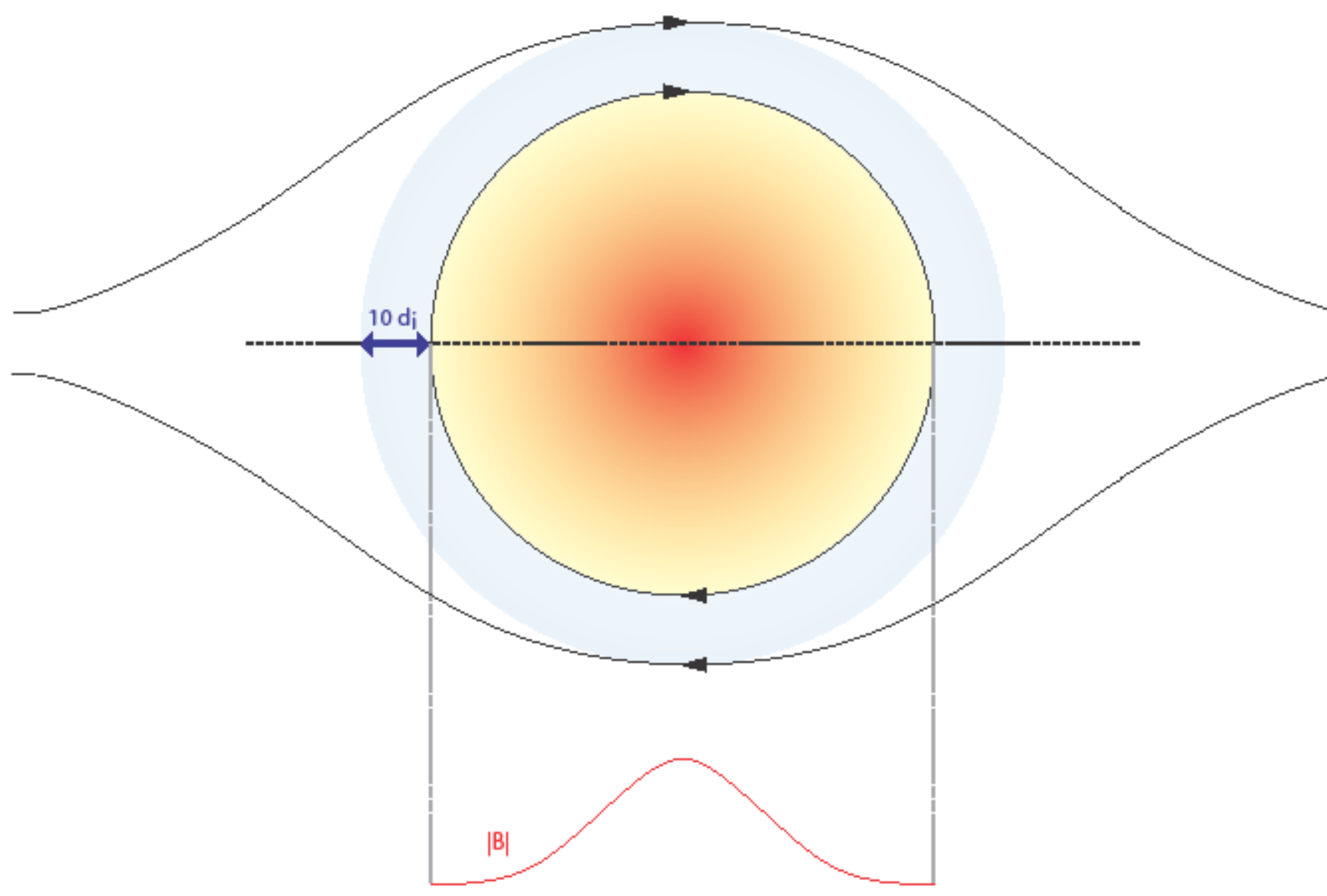

Figure 6: The red gradient indicates the magnetic field magnitude inside the FTE, while the light blue shade denotes the outer perimeter of the FTE with a thickness of 10 ion inertial lengths.

This article is protected by copyright. All rights reserved. 


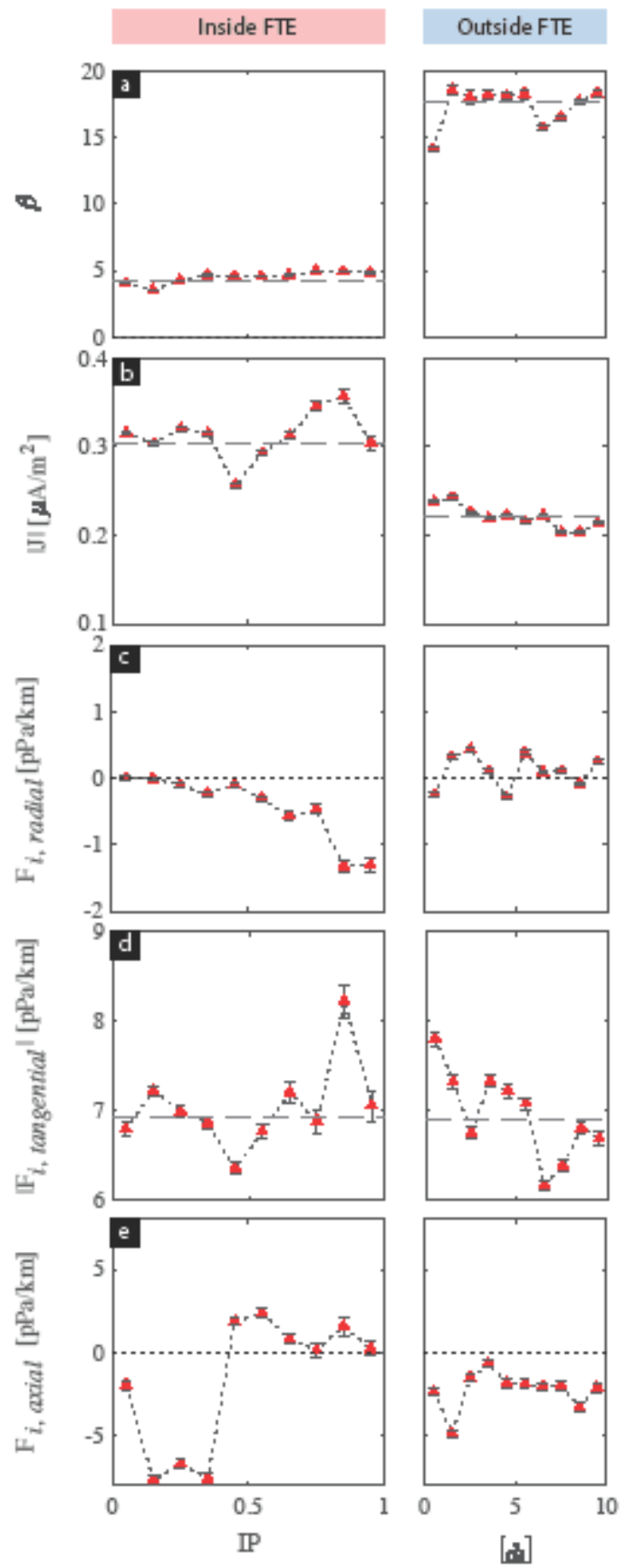

This article is protected by copyright. All rights reserved. 
Figure 7: The cross-sectional profiles of a) plasma beta, b) current density, $c$ ) the radial component of the net force, $d$ ) the magnitude of the tangential component of the net force, and e) the axial component of the net force inside and outside (10 local ion inertial lengths from the FTE edge) the 55 FTEs. All data points inside (outside) the 55 FTEs are grouped and averaged inside bins $\left(B W=1 d_{i}\right)$. The error bar denotes the standard error inside each individual bin.

This article is protected by copyright. All rights reserved. 

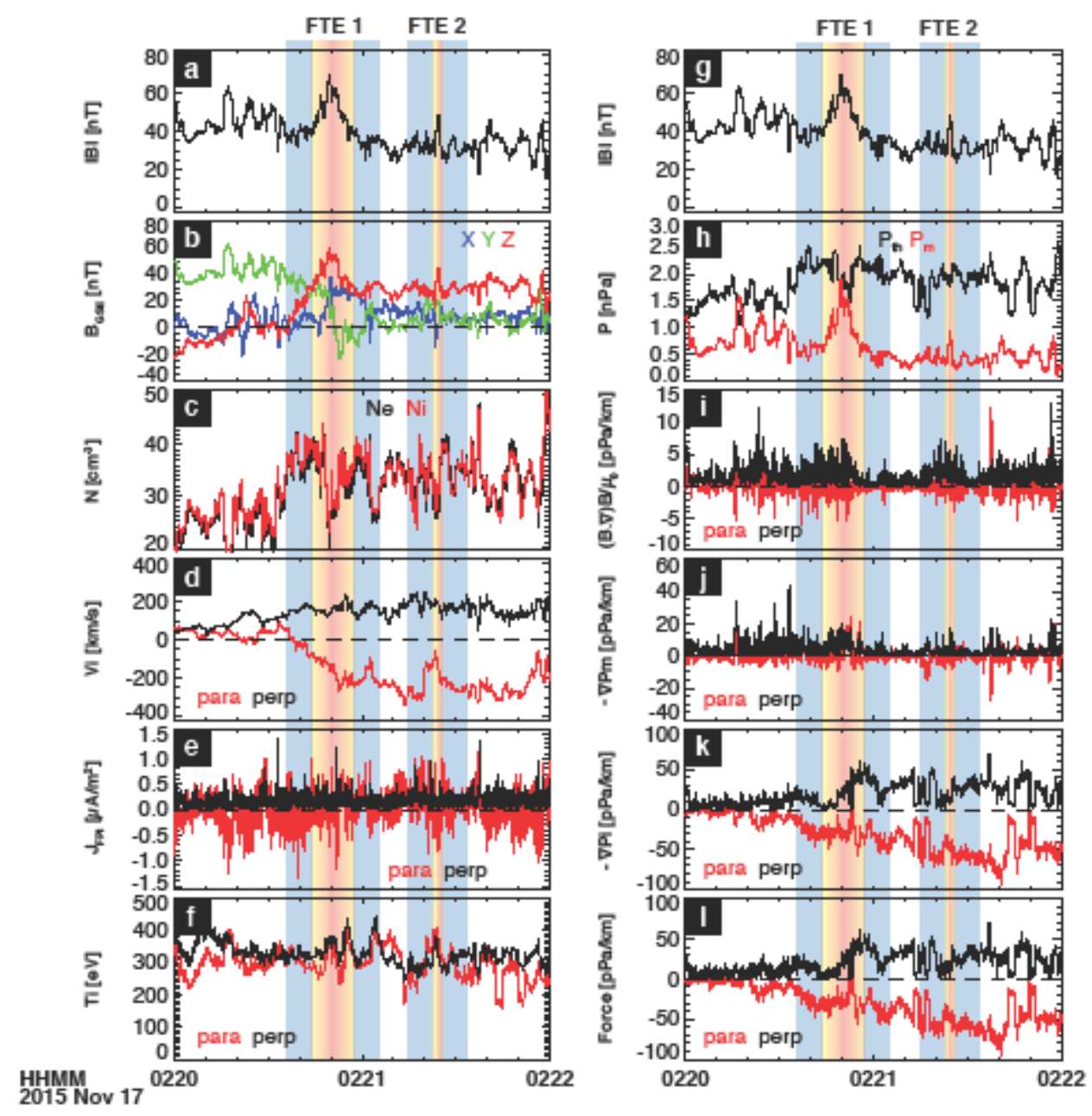

This article is protected by copyright. All rights reserved. 
Figure 8: The November 17, 2015 magnetopause boundary crossing. Panels include: a\&g) magnetic field magnitude, b) magnetic field components in the GSE coordinates, $c$ ) ion (red) and electron (black) density, $d$ ) ion velocity parallel (red) and perpendicular (black) components, $e$ ) plasma current density parallel and perpendicular components, $f$ ) ion temperature components, $h$ ) ion thermal and magnetic (black) pressures, $i$ ) magnetic curvature force parallel and perpendicular components, $j$ ) magnetic pressure gradient force parallel and perpendicular components, $k$ ) ion thermal pressure gradient force parallel and perpendicular components, and l) net force parallel and perpendicular components. Two quasi-force-free FTEs, labeled as 'FTE 1 ' and 'FTE 2,' are shown with gradient and solid color shadings corresponding to the observations made 'inside' and 'outside' the FTEs, respectively. The width of the blue solid color shading is 10 ion inertial lengths, $d_{i} \sim 1400 \mathrm{~km}$, from an average of $N_{i}=40 \mathrm{~cm}^{-3}$. 

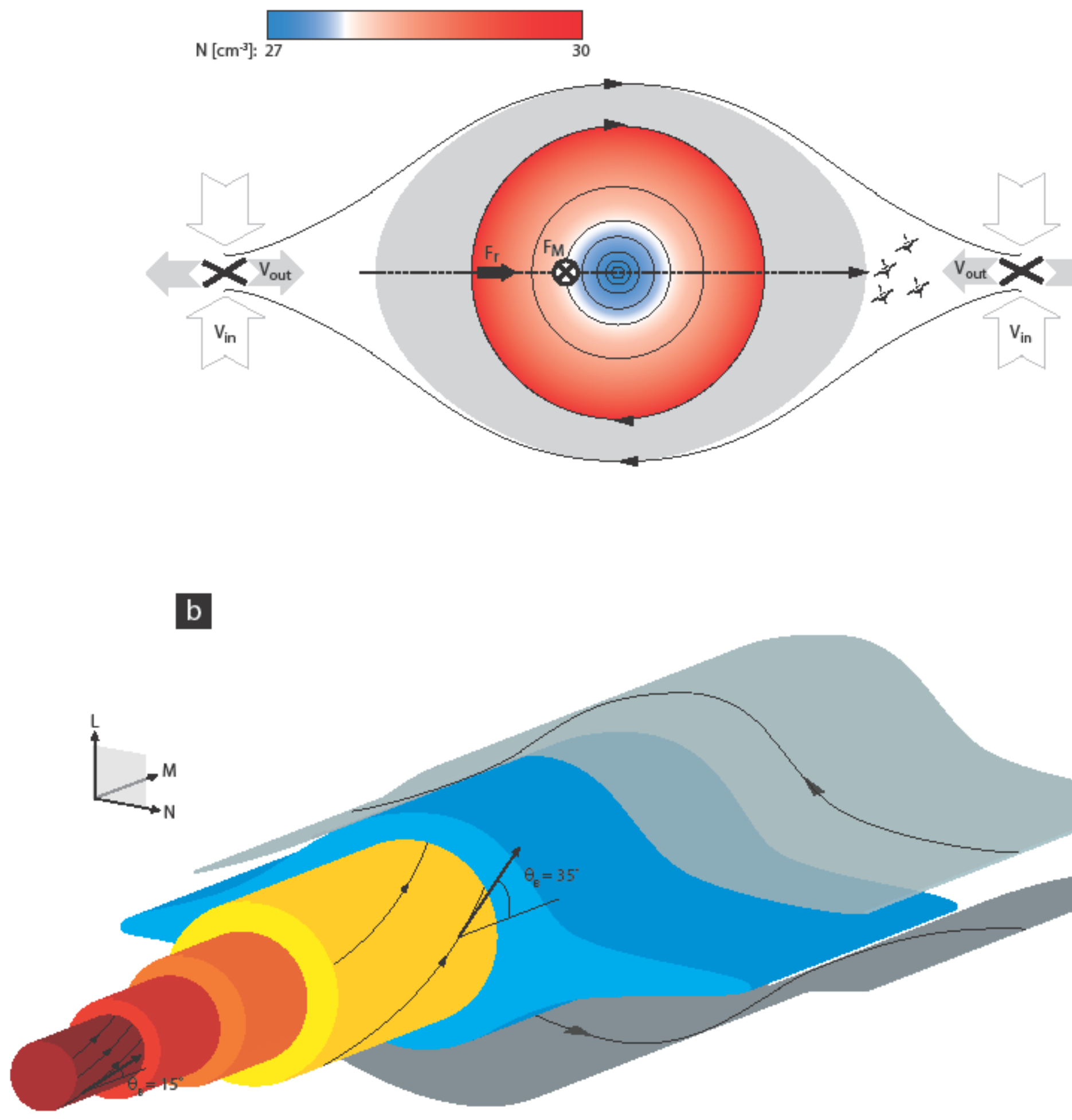

This article is protected by copyright. All rights reserved. 
Figure 9: Cartoon representation of cross-sectional profiles of a) plasma density profile and the radial (solid black arrow) and the axial (into-the plane arrow) components of the net force, and b) magnetic pitch angle, $\theta_{B}=$ atan $\left(B_{L} / B_{M}\right)$, where $B_{L}$ and $B_{M}$ represent tangential and axial magnetic field components, respectively. Plasma density colorbar corresponds to the binaveraged values reported in this study. The grey shaded area around the flux rope illustrates the magnetic flux and plasma (grey arrows labelled as ' $V_{\text {out }}$ ') continuously added to the FTEs' outer layers by reconnection (shown as ' $X$ '). 

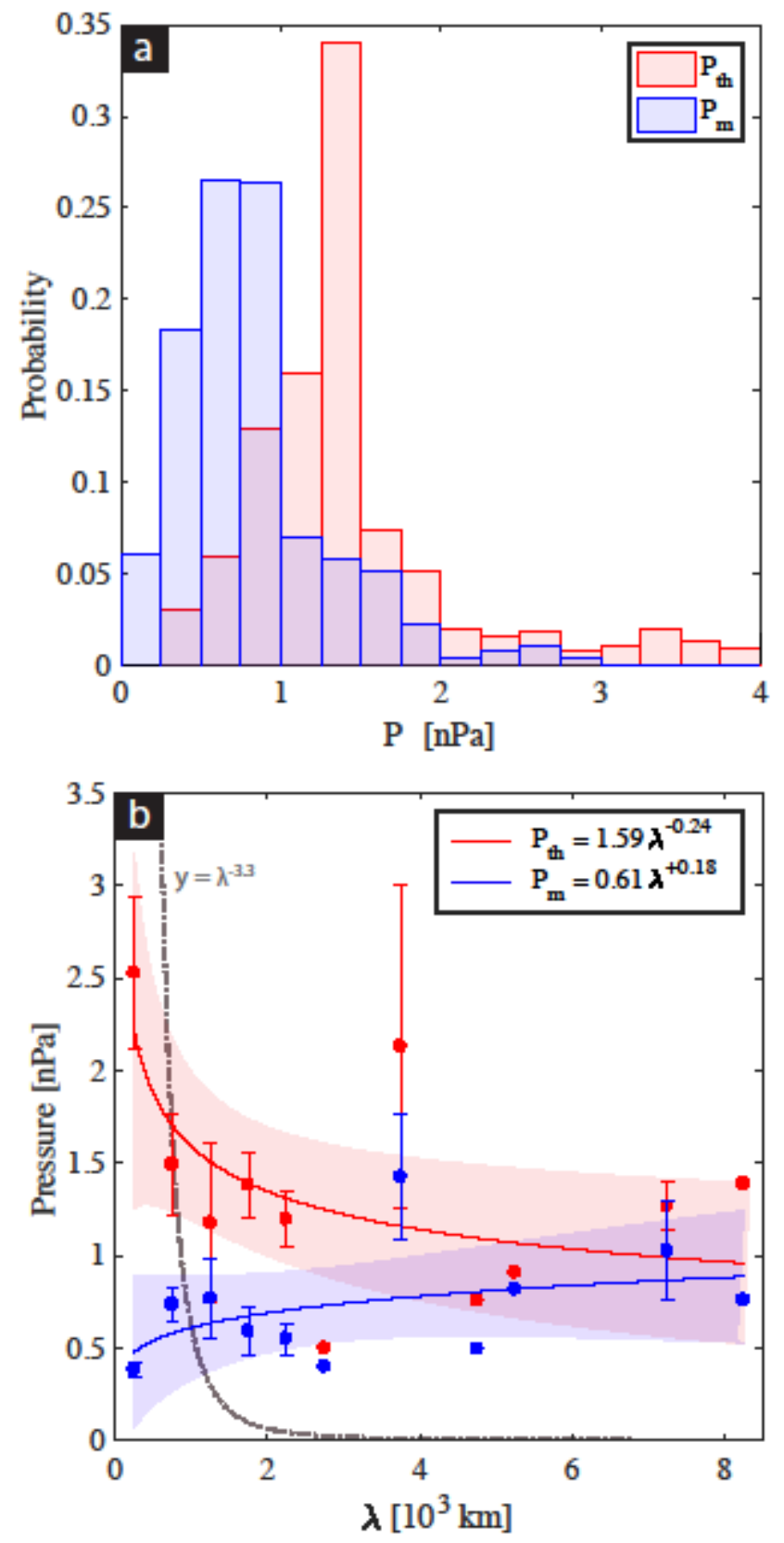

This article is protected by copyright. All rights reserved. 
Figure 10: a) Histograms of thermal and magnetic pressure data points inside the 55 FTEs. $b$ ) Average thermal pressure and magnetic pressure profiles as a function of FTE diameter across the 55 FTEs. The circles indicate the bin-averaged $(B W=500 \mathrm{~km})$ values of pressures. 


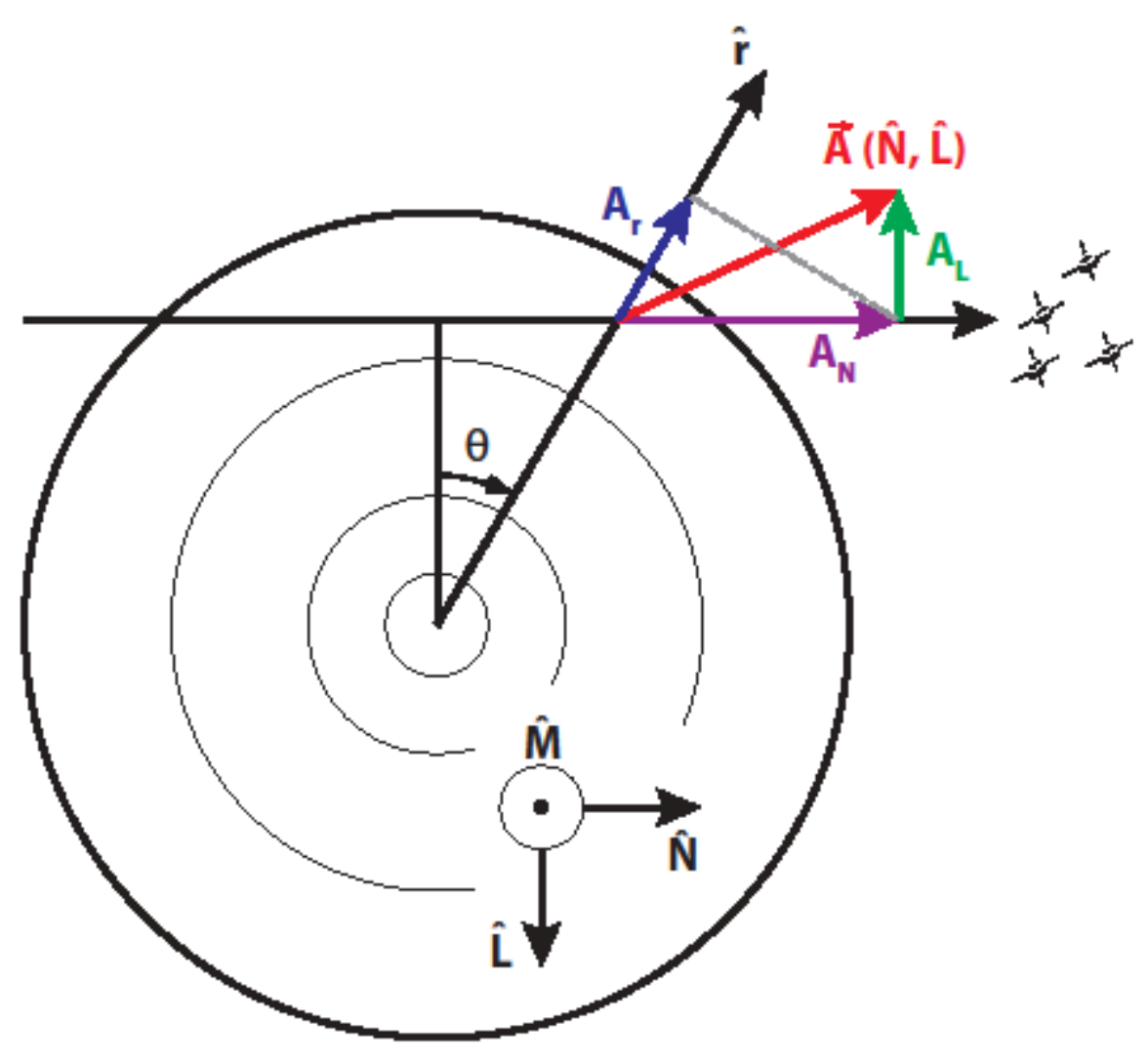

This article is protected by copyright. All rights reserved. 
Figure 11: Coordinate transformation between flux rope's LMN and cylindrical coordinate systems.

Table I: Thermal $\left(P_{t h}\right)$ and magnetic $\left(P_{m}\right)$ pressure averages inside and outside FTEs observed in the magnetosheath (MSH) and at the magnetopause (MP). The ratio of pressures inside and outside FTEs (ratio $\left.=P_{\text {inside }} / P_{\text {outside }}\right)$ is determined for each category.

Table II: The relative contribution, i.e., the percentage of total, of the force terms inside (blue shade) and outside (red shade) FTEs. The shading in each row corresponds to the relative contribution to the total force average inside all 55 FTEs with the darkest shading representing the largest contribution. 


\section{Pressure-Imbalance-Driven Expansion}

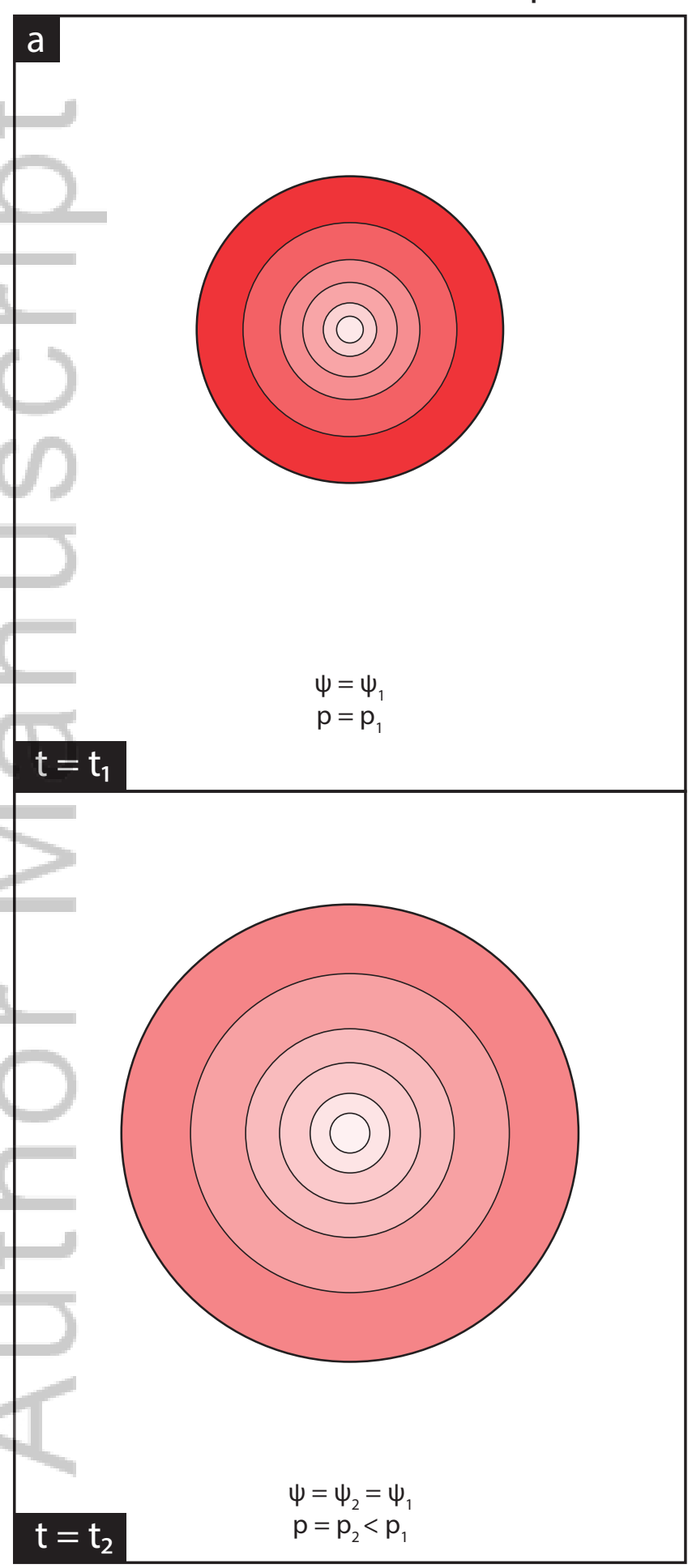

Continuous Reconnection

b

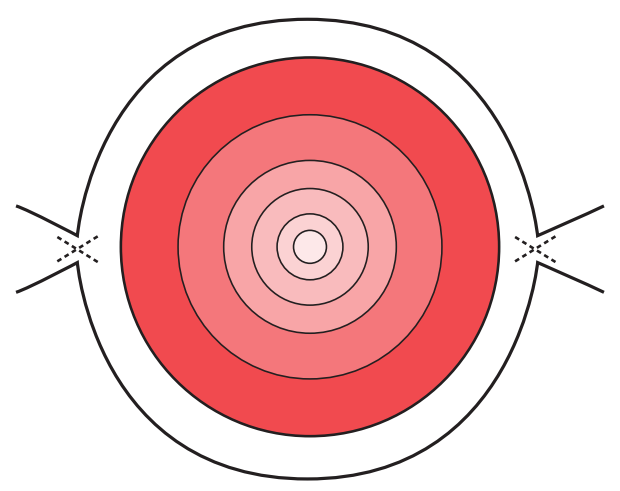

$\psi=\psi_{1}$

\section{$t=t_{1}$}

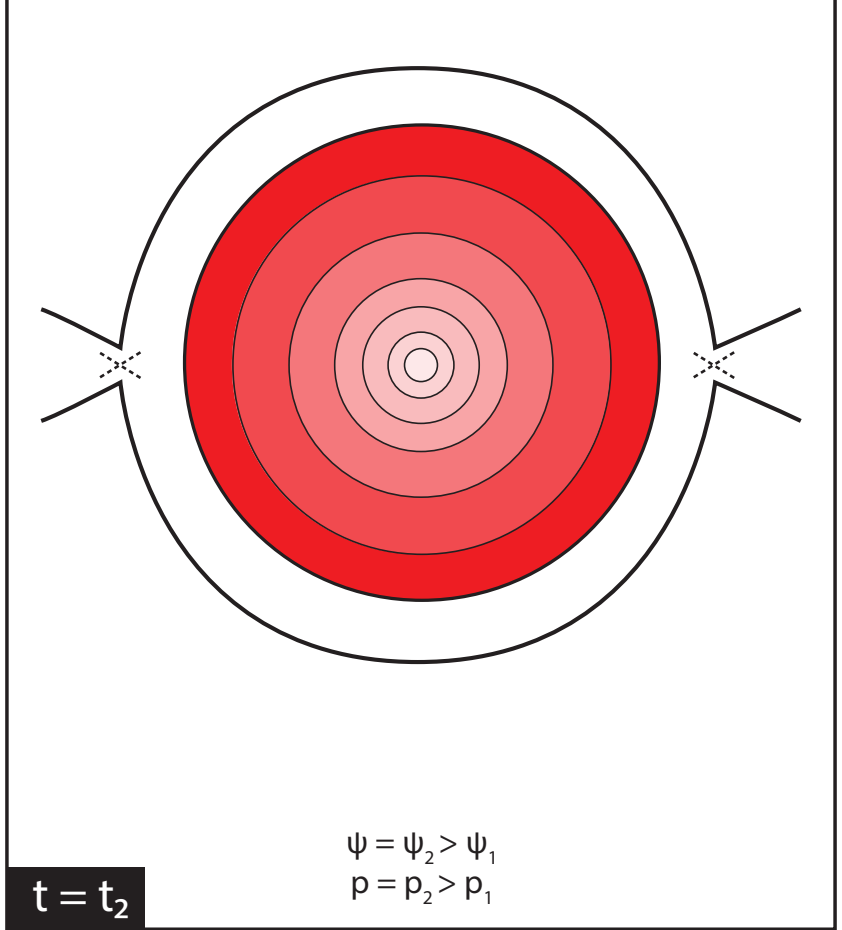

Secondary Reconnection (Coalescence)

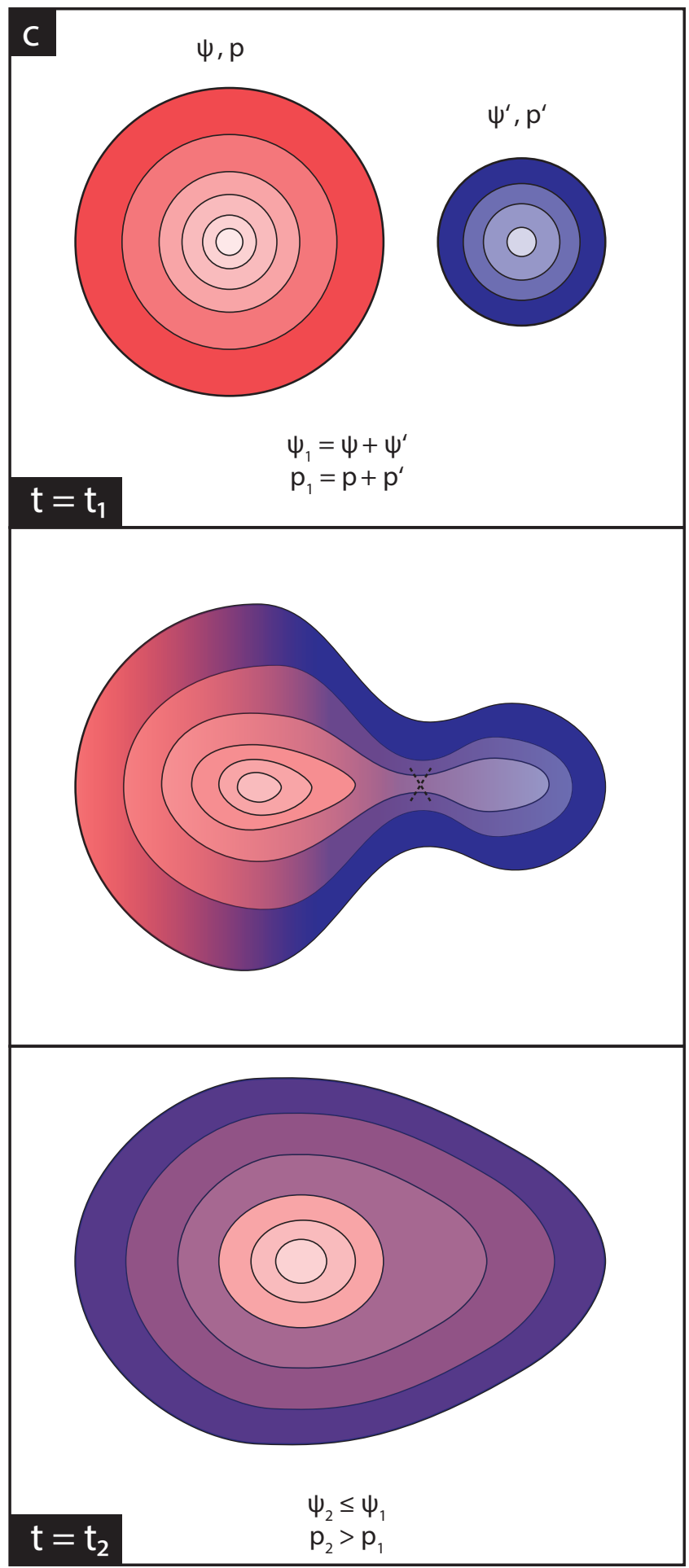

This article is protected by copyright. All rights reserved. 

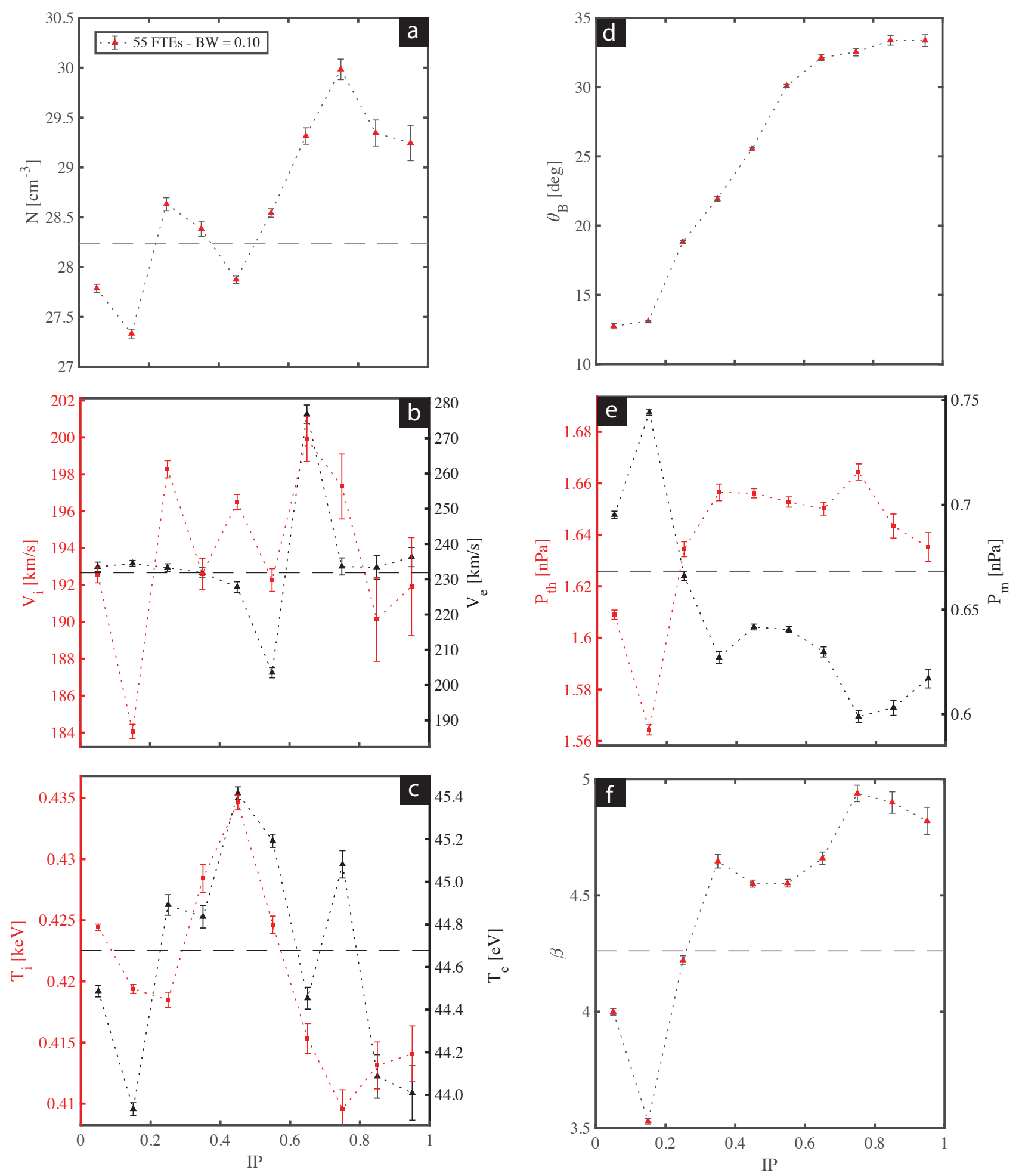

This article is protected by copyright. All rights reserved. 

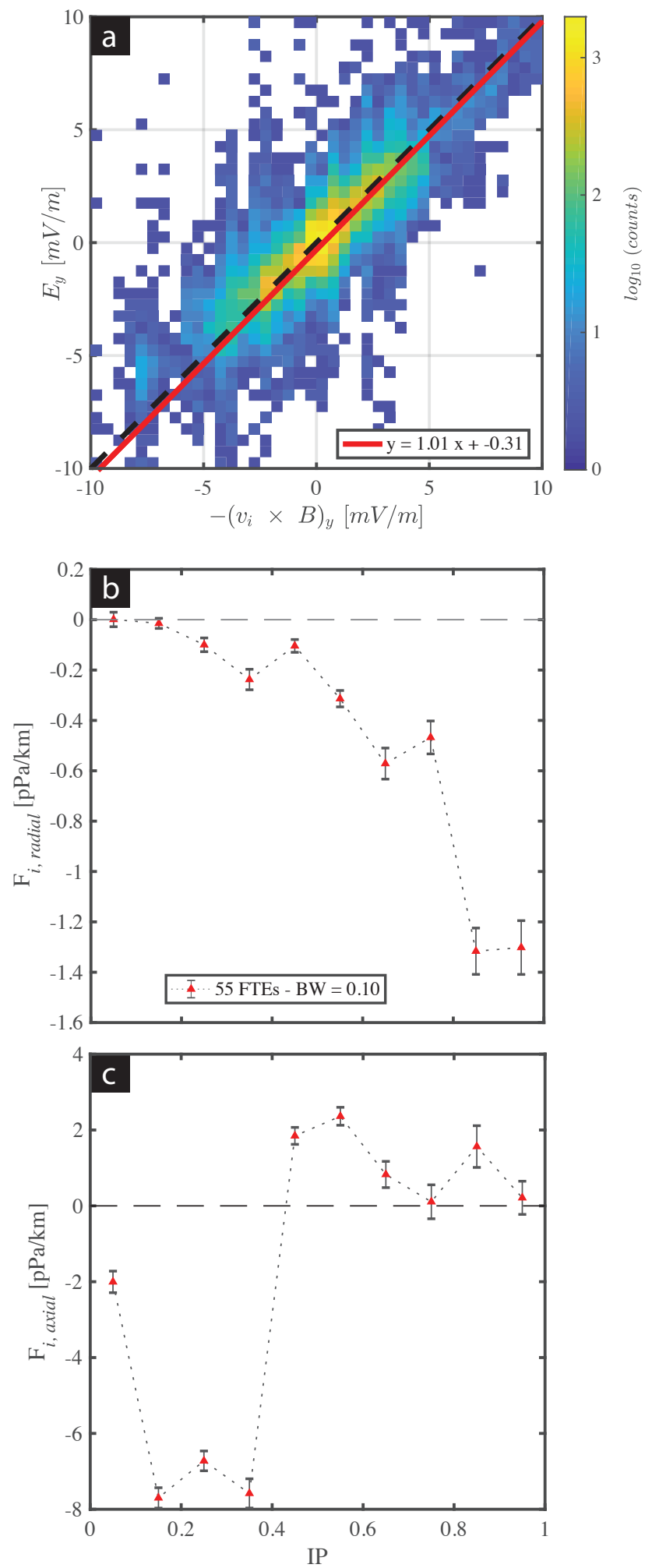

This article is protected by copyright. All rights reserved. 


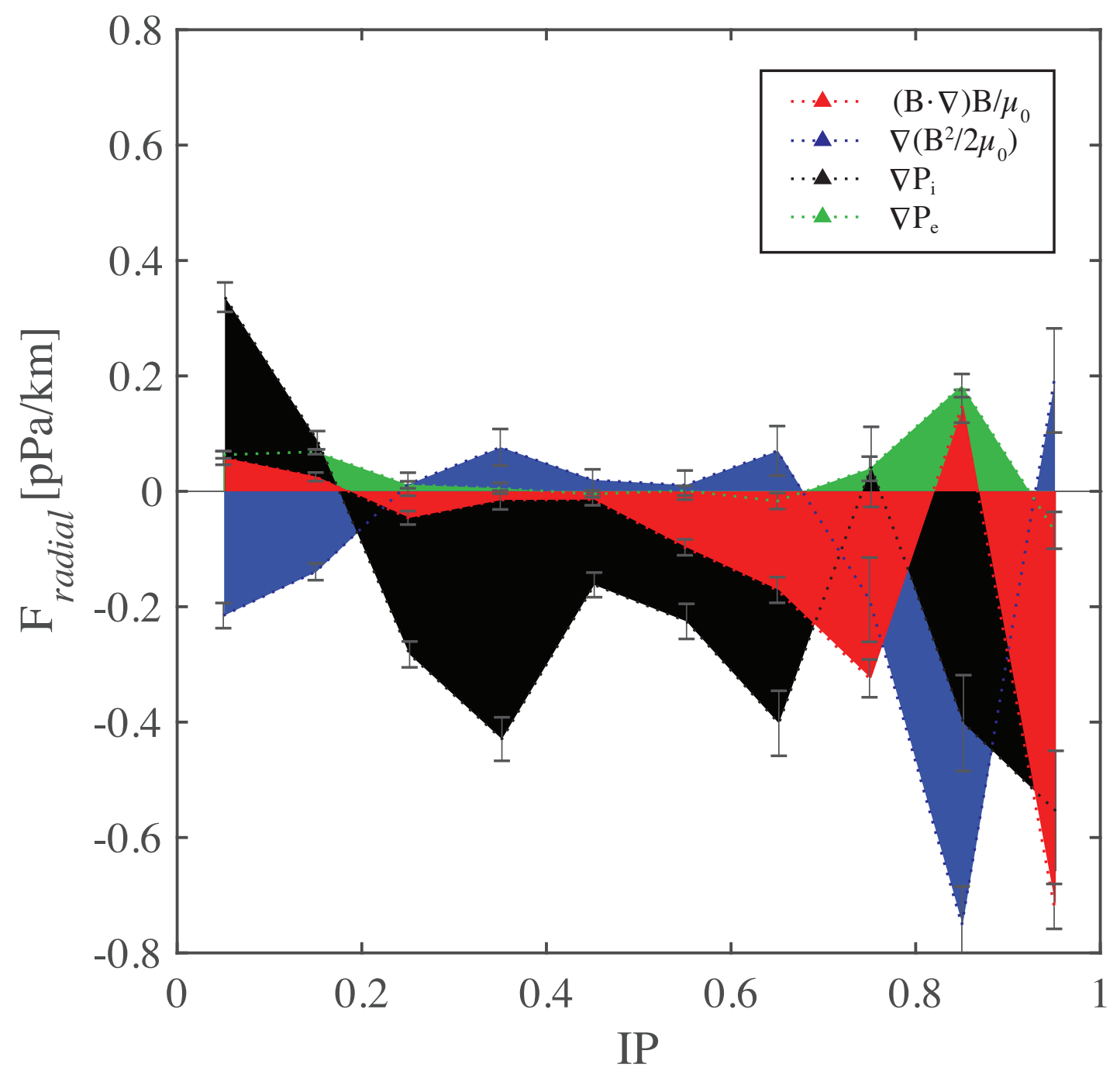

This article is protected by copyright. All rights reserved. 

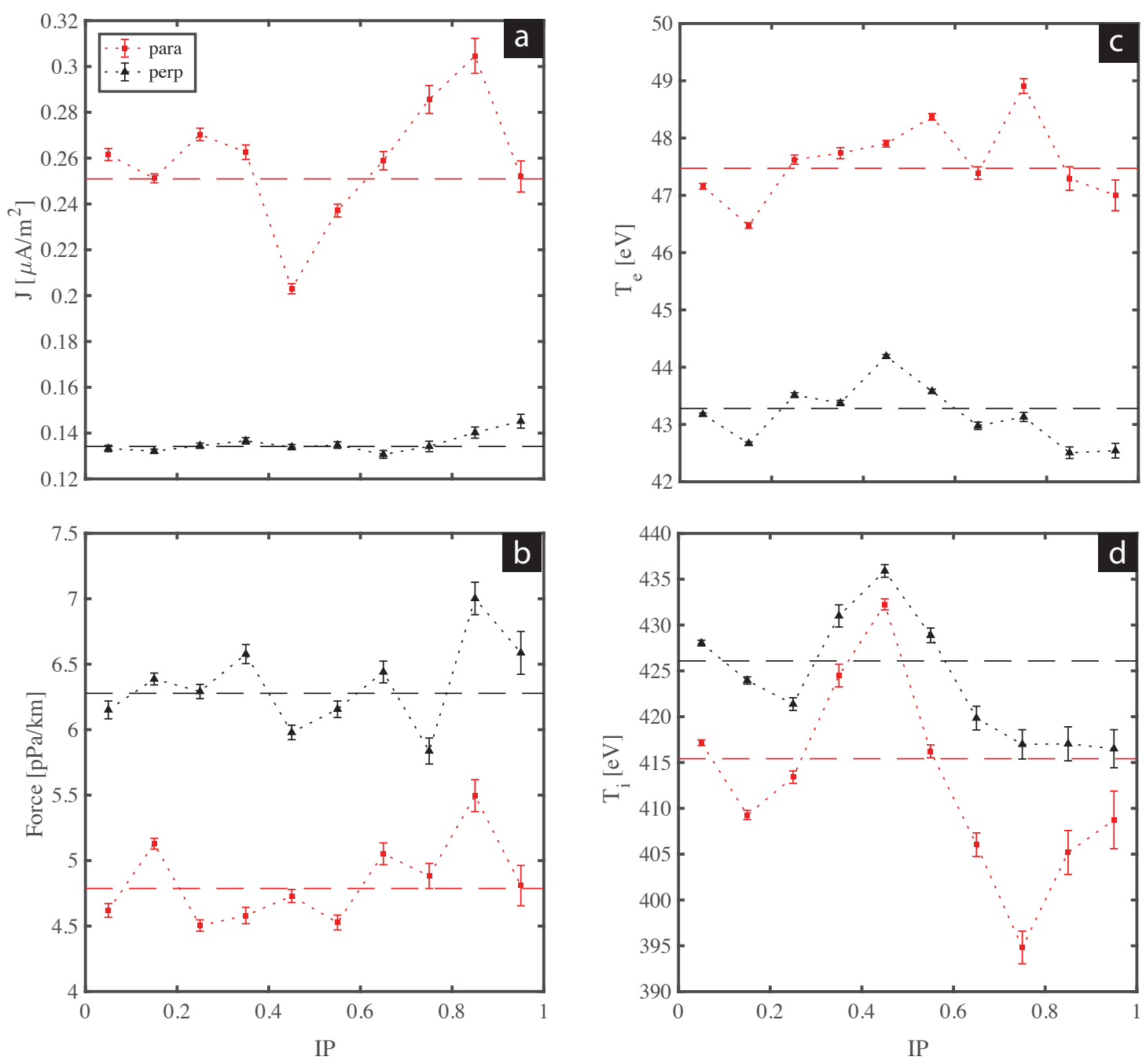

This article is protected by copyright. All rights reserved. 


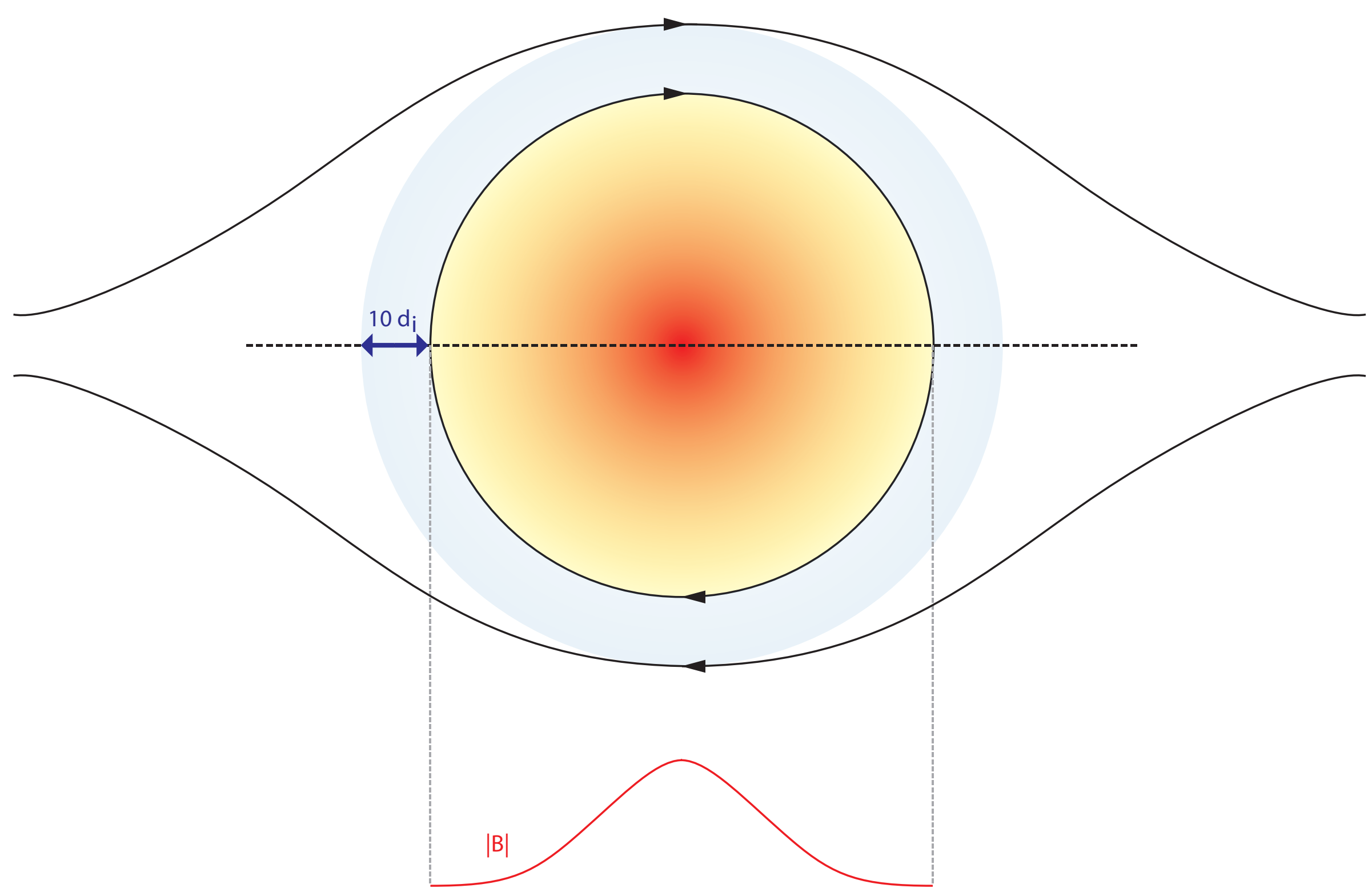

This article is protected by copyright. All rights reserved. 

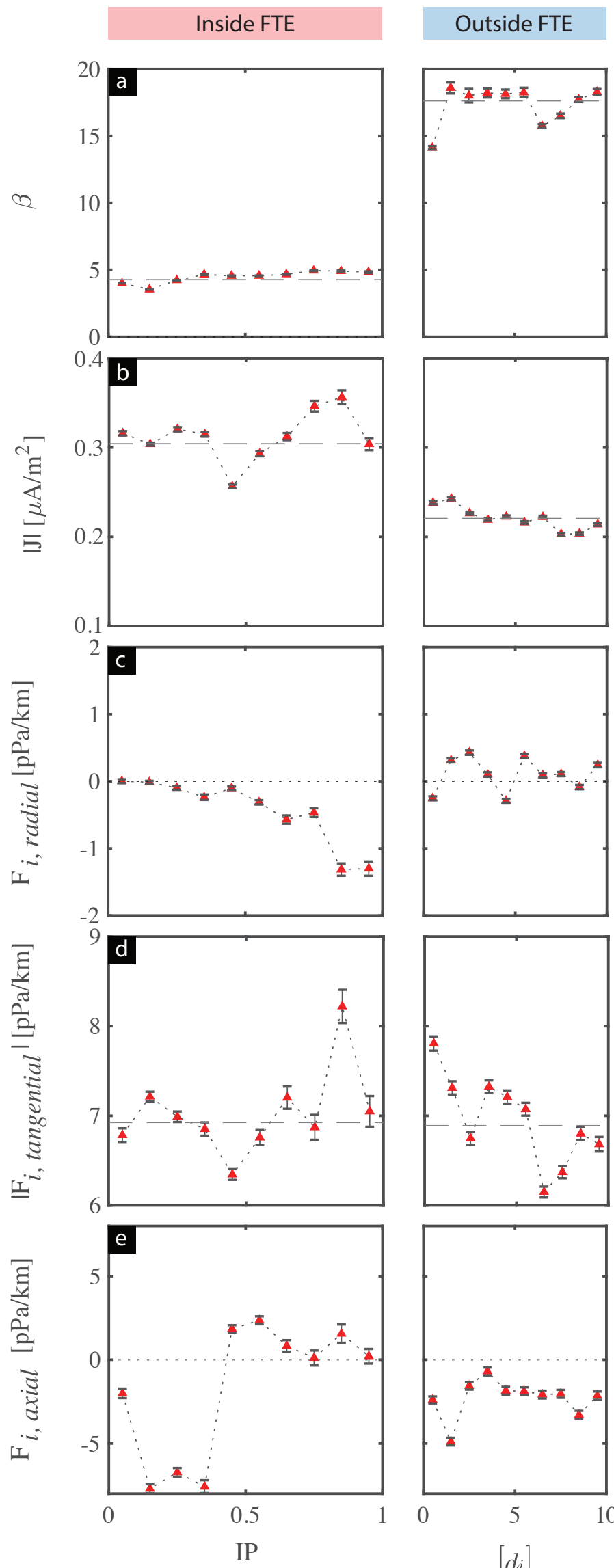

This article is protected by copyright. All rights reserved. 
FTE 1 FTE 2
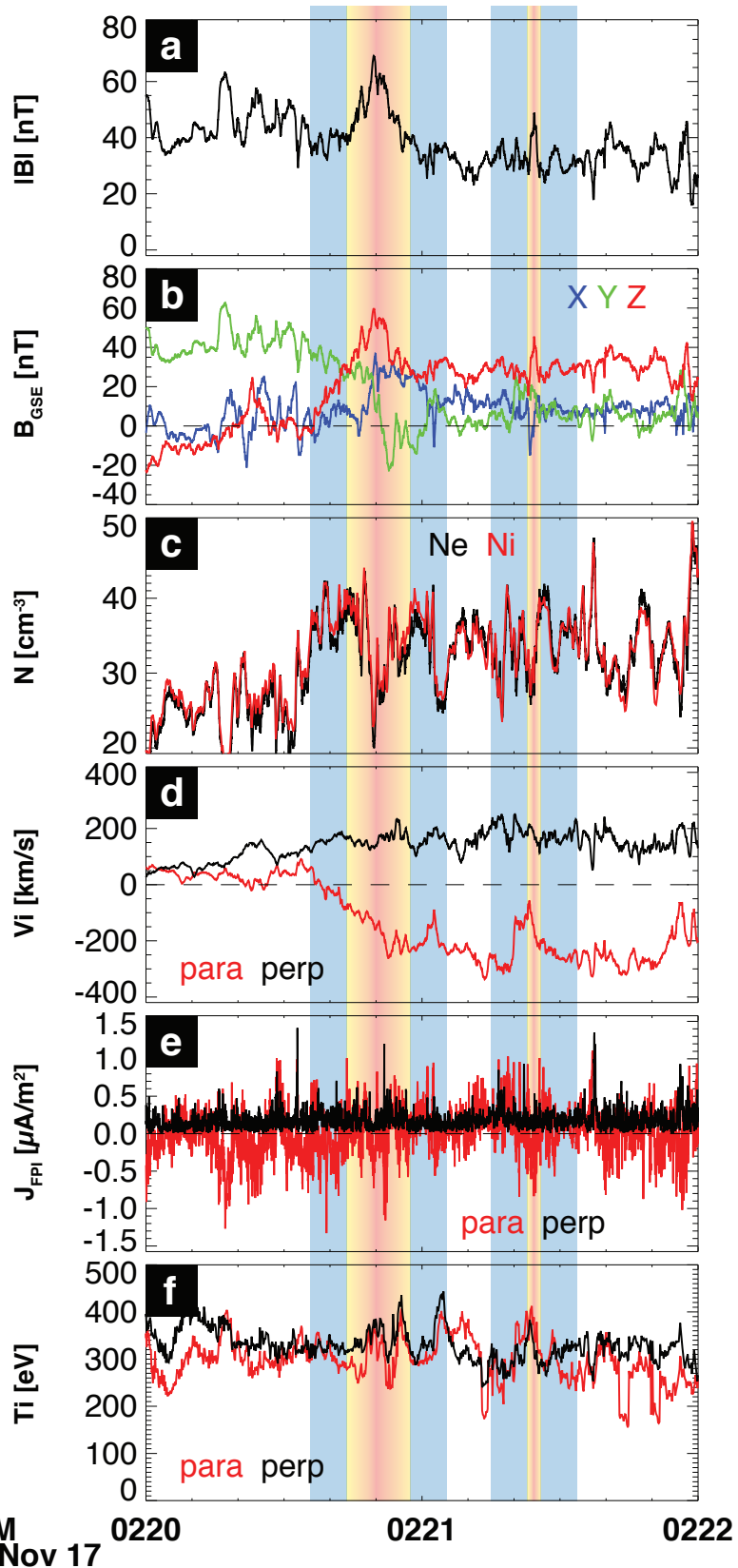

FTE 1 FTE 2

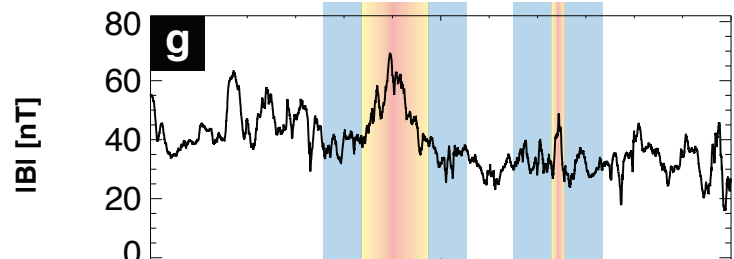

$3.0 \mathrm{~h}$

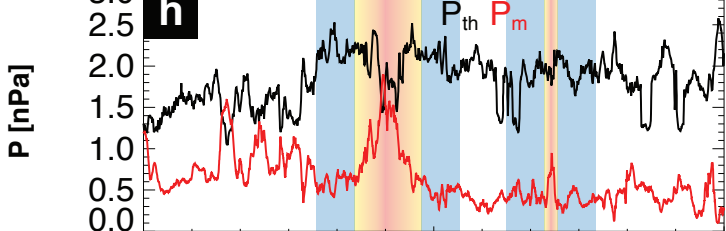

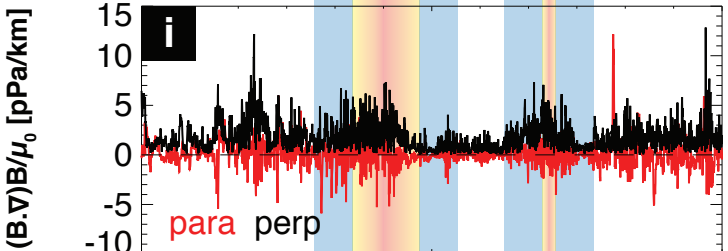

${ }_{40}^{60} \mathbf{j}$
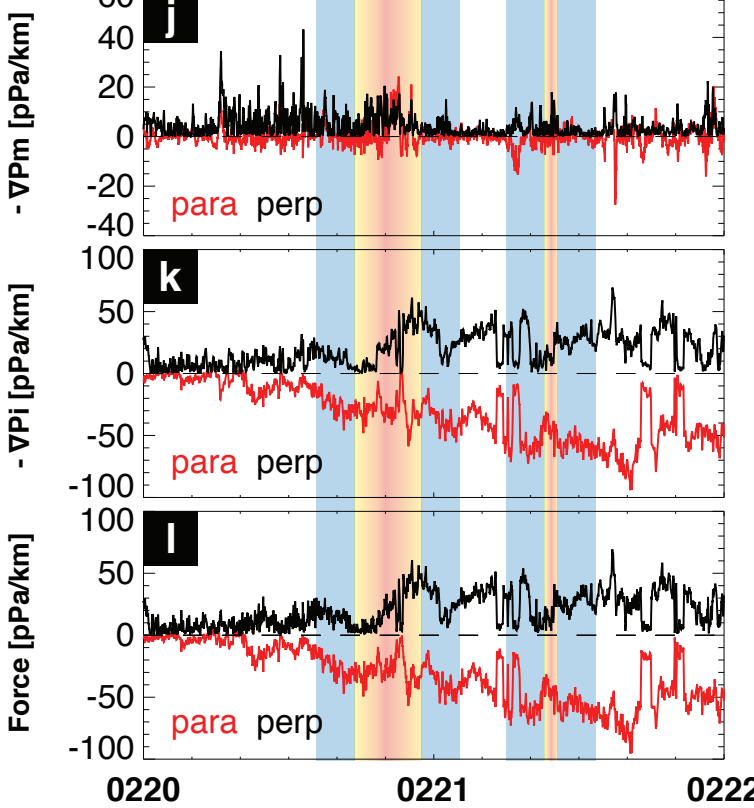
$\mathrm{N}\left[\mathrm{cm}^{-3}\right]: 27$

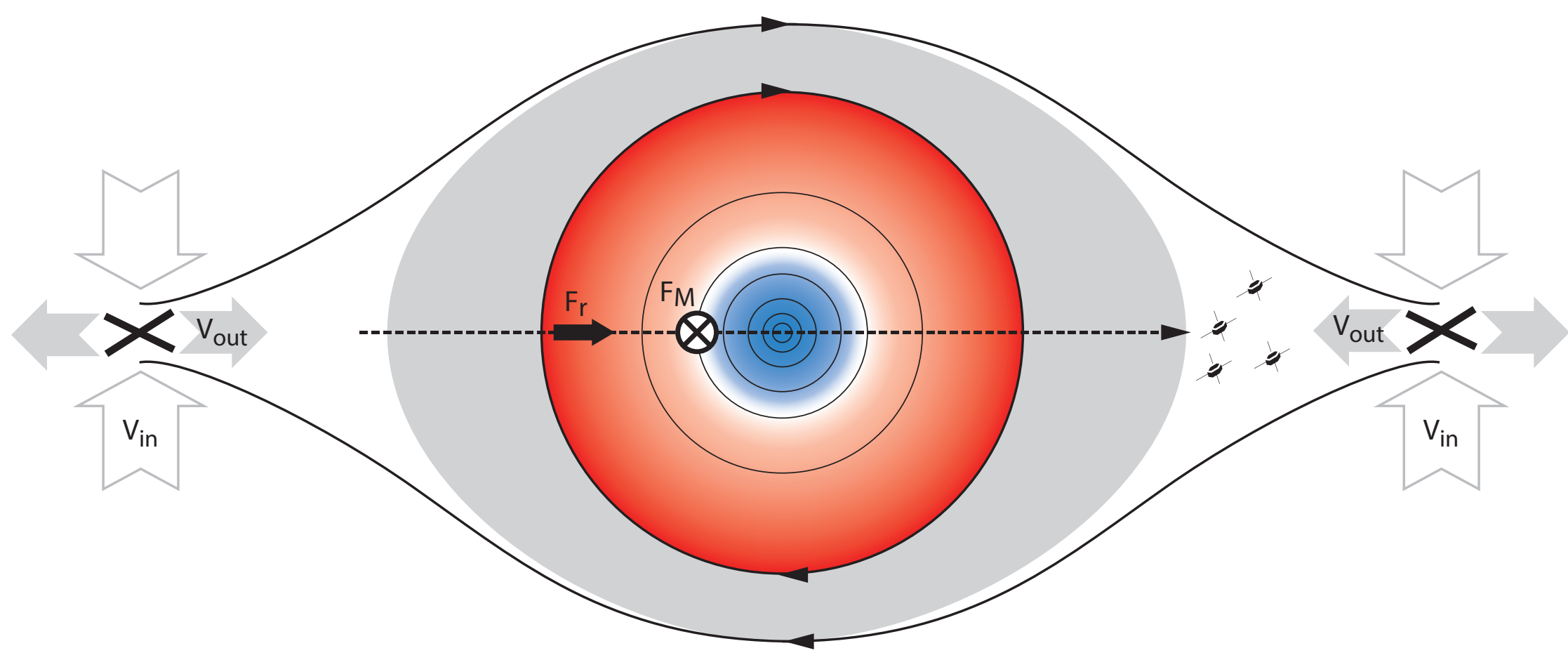

b
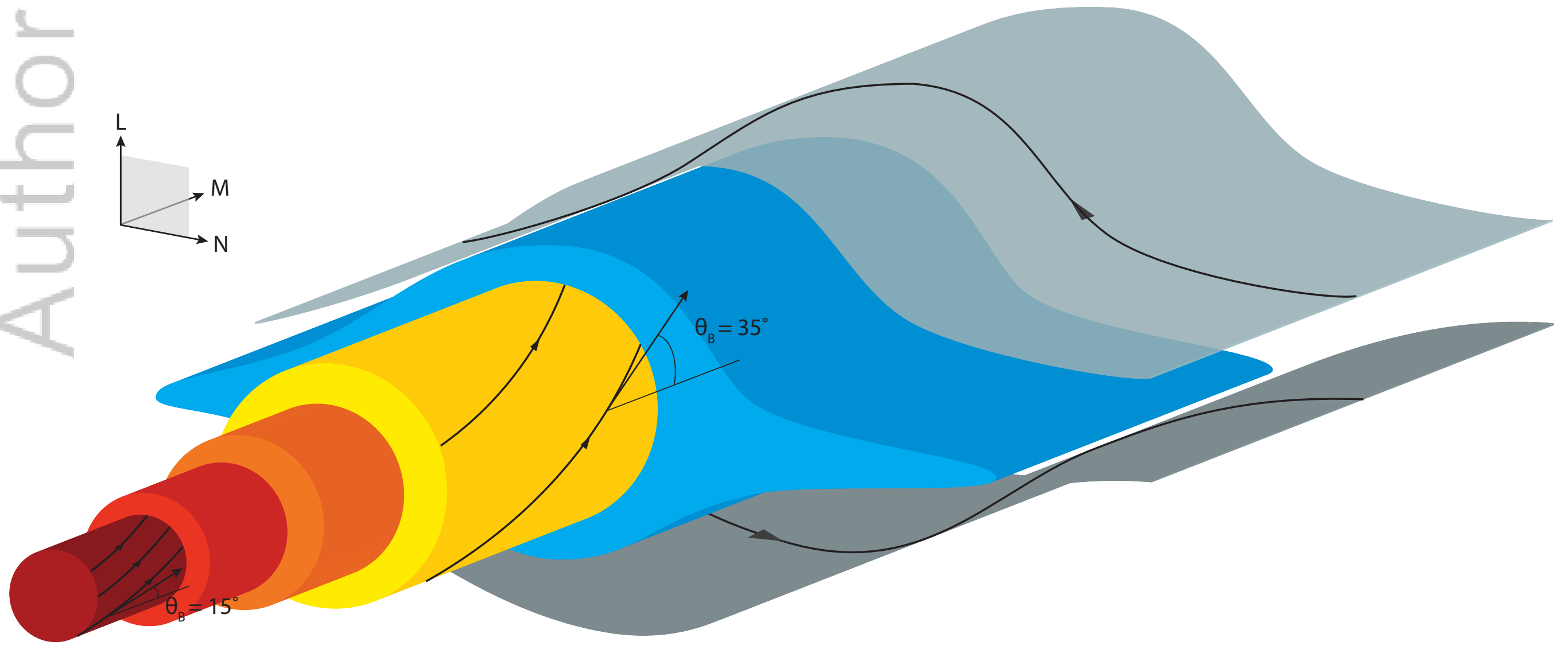

This article is protected by copyright. All rights reserved. 

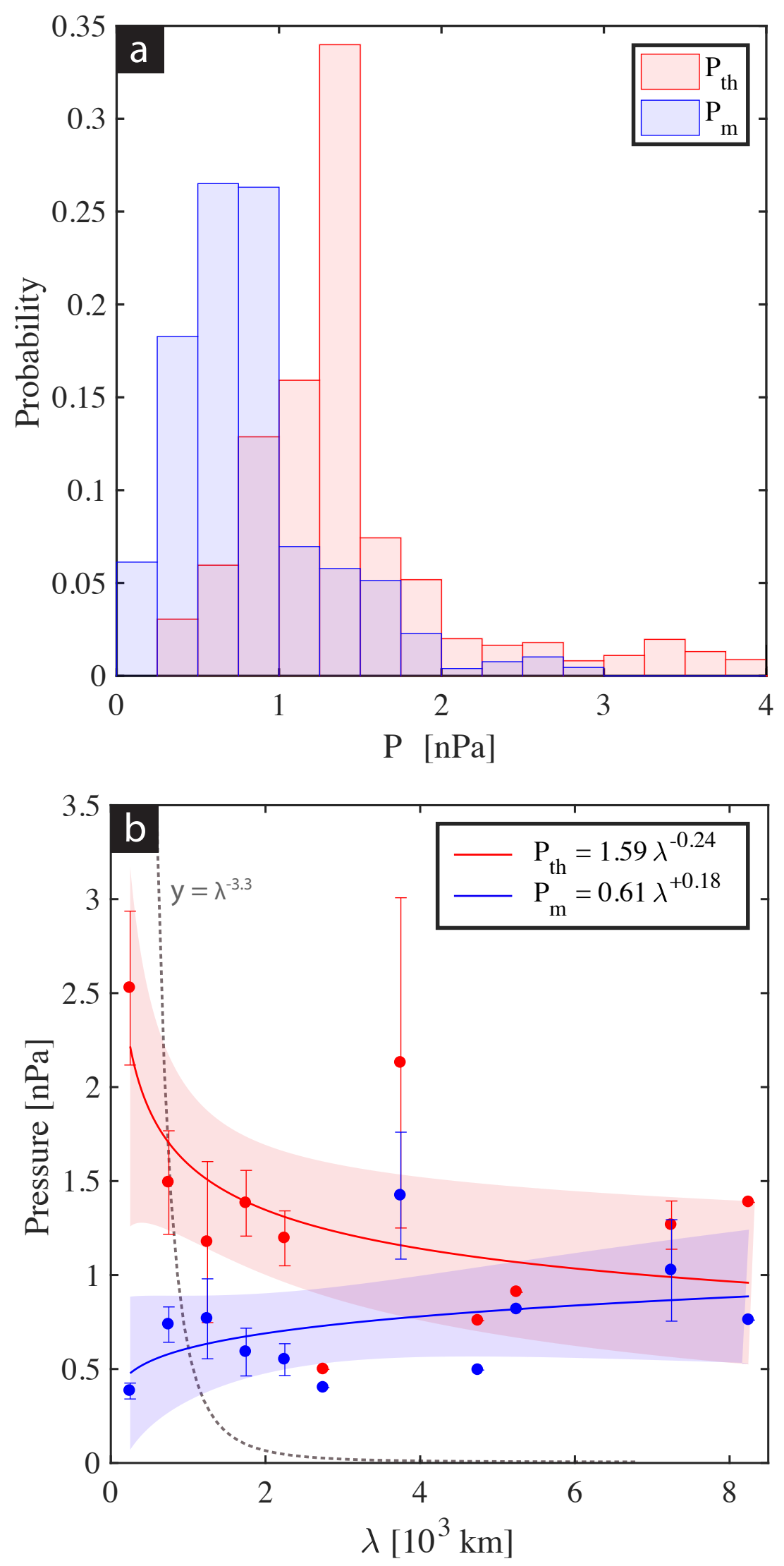

This article is protected by copyright. All rights reserved. 


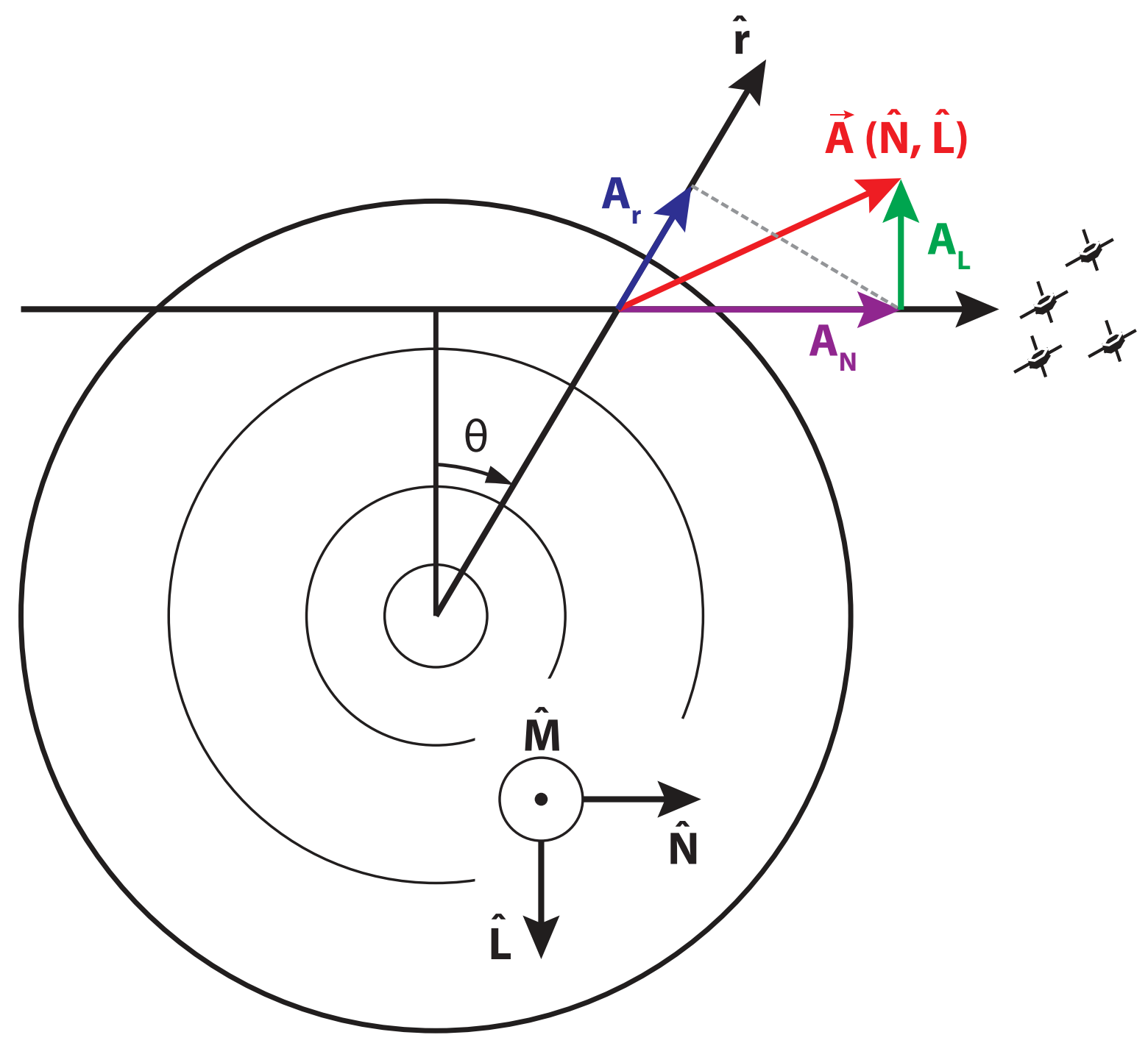

This article is protected by copyright. All rights reserved. 